\title{
FAMILY OF ESTIMATORS OF MEAN, RATIO, AND PRODUCT OF A FINITE POPULATION USING RANDOM NONRESPONSE
}

\author{
Housila P. Singh \\ School of Studies in Statistics \\ Vikram University \\ Ujjain, M.P. 456010, India \\ e-mail: hpsujn@rediffmail.com \\ Prem Chandra \\ Institute for research in Medical Statistics \\ ICMR, New Delhi-110029, India \\ Anwar H. Joarder \\ Department of Mathematical Sciences \\ King Fahd University of Petroleum \& Minerals \\ Dhahran 31261, Saudi Arabia \\ e-mail: anwarj@kfupm.edu.sa \\ Sarjinder Singh \\ Department of Statistics \\ St. Cloud State University \\ St. Cloud, MN 56301-4498, USA \\ e-mail: sarjinder@yahoo.com
}

\begin{abstract}
In this paper a family of estimators for estimating mean, ratio and product of two means of a finite population has been suggested and studied under the two different situations of random non-response considered by Tracy and Osahan (1994), Singh and Joarder (1998) and Singh, Joarder and Tracy (2000). Asymptotic expressions of biases and mean squared errors of the proposed families have been derived. Optimum conditions have been obtained under which the proposed families of estimators have the minimum mean squared error (MSE). Further the optimum values (depending upon population parameters) when replaced by sample values yield the estimators having the minimum MSE of the optimum estimators. The estimators for MSE's of the suggested families are also given.
\end{abstract}

Keywords: Auxiliary information; random non-response; bias; mean squared error. 


\section{Introduction}

In sample surveys it is common to make use of auxiliary information to increase the precision of the estimates of population parameters. The problem of estimating the ratio and product of two means of a finite population using information on single (or more) auxiliary variables has been discussed among others, by Singh (1965, 1967), Rao and Pereira (1968), Shah and Shah (1978), Tripathi (1980), Ray and Singh (1985), Upadhyaya and Singh (1985), Upadhyaya et. al. (1985), Singh (1986a, 1986b, 1988), Srivastava et al. (1989) and Singh et al. (1994a, 1994b). Let $y_{i}(i=0,1)$ be the study characters with population mean $\bar{Y}_{i}(i=0,1)$ and $y_{2}$ be the auxiliary character (correlated with study characters $y_{i}(i=0,1)$ with known population mean $\bar{Y}_{2}$. Assume that a simple random sample of size $n$ is drawn without replacement and $\left(y_{0 i}, y_{l i}, y_{2 i}\right), i=1,2, \ldots, n$ are observed. The usual estimator of ratio $R_{(\alpha)}=\bar{Y}_{0} / \bar{Y}_{1}^{\alpha}\left(\bar{Y}_{1} \neq 0\right)$ is defined by

$$
\hat{R}_{(\alpha)}=\frac{\bar{y}_{0}}{\bar{y}_{1}^{\alpha}},\left(\bar{y}_{1} \neq 0\right)
$$

where $\alpha$ is a scalar which takes value 0,1 and -1 . It is to be mentioned that:

(i) for $\alpha=0, R_{(\alpha)} \rightarrow R_{(0)}=\bar{Y}_{0}$ and its estimator $\hat{R}_{(\alpha)} \rightarrow \hat{R}_{0)}=\bar{y}_{0}$,

(ii) for $\alpha=1, R_{(a)} \rightarrow R_{(1)}=\bar{Y}_{0} / \bar{Y}_{1}$ and its estimator $\hat{R}_{(\alpha)} \rightarrow \hat{R}_{(1)}=\bar{y}_{0} / \bar{y}_{1}=\hat{R}$,

(iii) for $\alpha=-1, R_{(a)} \rightarrow R_{(-1)}=\bar{Y}_{0} \bar{Y}_{1}$ and its estimator $\hat{R}_{(\alpha)} \rightarrow \hat{R}_{(-1)}=\bar{y}_{0} \bar{y}_{1}=$ $\hat{P}$.

Utilizing the information on an auxiliary character $y_{2}$, a class of estimators for $R_{(\alpha)}$ on the basis of Srivastava (1971) is defined by

$$
\hat{R}_{(\alpha)}^{(h)}=\hat{R}_{(\alpha)} h(u)
$$

where $h(u)$ is a function of $u=\bar{y}_{2} / \bar{Y}_{2}$ such that $h(1)=1$ and satisfies certain regularity conditions as mentioned in Srivastava (1971). Motivated by Srivastava (1980), we propose a wider class of estimators for $R_{(\alpha)}$ as

$$
\hat{R}_{(\alpha)}^{(H)}=H\left(\hat{R}_{(\alpha)}, u\right)
$$

where $H\left(\hat{R}_{(\alpha)}, u\right)$ is a function of $\left(\hat{R}_{(\alpha)}, u\right)$ such that $H\left(R_{(\alpha)}, 1\right)=R_{(\alpha)}, H_{1}\left(R_{(\alpha)}, 1\right)=$ 1 and satisfies certain conditions as given in Srivastava (1980).

Assuming the population mean $\bar{Y}_{2}$ and variance $S_{2}^{2}$ of the auxiliary character $y_{2}$ to be known and following the same approach as adopted by Srivastava and 
Jhajj (1981) and Upadhyaya and Singh (1985), a family of estimators of $R_{(\alpha)}$ is defined by

$$
\hat{R}_{(\alpha)}^{(t)}=\hat{R}_{(\alpha)} t(u, v)
$$

where $t(u, v)$ is a function of $(u, v)$ such that $t(1,1)=1$ and satisfies certain regularity conditions as defined in the Appendix. For $\alpha=1, \hat{R}_{(\alpha)}^{(t)}$ reduces to Upadhyaya and Singh (1985) estimator of ratio $R_{(1)}=R$ given by

$$
\hat{R}_{(1)}^{(t)}=\hat{R} t(u, v)
$$

while for $\alpha=0$, it reduces to Srivastava and Jhajj (1981) estimator of population mean $\bar{Y}_{0}$

$$
\hat{R}_{(0)}^{(t)}=\bar{y}_{0} t(u, v)
$$

To the first degree of approximation, the biases and MSE's of $\hat{R}_{(\alpha)}^{(h)}$ and $\hat{R}_{(\alpha)}^{(t)}$ are respectively given by

$$
\begin{gathered}
B\left(\hat{R}_{(\alpha)}^{(h)}\right)=B\left(\hat{R}_{(\alpha)}\right)+\left(\frac{\theta R_{(\alpha)}}{2}\right) C_{2}^{2}\left\{2 K_{(\alpha)} h_{1}(1)+h_{11}(1)\right\} \\
B\left(\hat{R}_{(\alpha)}^{(t)}\right)=B\left(\hat{R}_{(\alpha)}\right)+\left(\frac{\theta R_{(\alpha)}}{2}\right)\left[2 K_{(\alpha)} C_{2}^{2} t_{1}(1,1)+2 d_{(\alpha)} t_{2}(1,1)\right. \\
\left.+C_{2}^{2} t_{11}(1,1)+2 \lambda_{003} C_{2} t_{12}(1,1)+\left(\lambda_{004}-1\right) t_{22}(1,1)\right] \\
\operatorname{MSE}\left(\hat{R}_{(\alpha)}^{(h)}\right)=\operatorname{MSE}\left(\hat{R}_{(\alpha)}\right)+\theta R_{(\alpha)}^{2} C_{2}^{2} h_{1}(1)\left\{h_{1}(1)+2 K_{(\alpha)}\right\}
\end{gathered}
$$

and

$$
\begin{aligned}
& M S E\left(\hat{R}_{(\alpha)}^{(t)}\right)=\operatorname{MSE}\left(\hat{R}_{(\alpha)}\right)+\theta R_{(\alpha)}^{2}\left[C_{2}^{2} t_{1}^{2}(1,1)+\left(\lambda_{004}-1\right) t_{2}^{2}(1,1)\right. \\
& \left.\quad+2 \lambda_{003} C_{2} t_{1}(1,1) t_{2}(1,1)+2 K_{(\alpha)} C_{2}^{2} t_{1}(1,1)+2 d_{(\alpha)} t_{2}(1,1)\right]
\end{aligned}
$$

where the bias and MSE of $\hat{R}_{(\alpha)}$, to the first degree of approximation, are respectively given by

$$
B\left(\hat{R}_{(\alpha)}\right)=\theta C_{1}^{2} R_{(\alpha)}(\alpha / 2)\left[\alpha-2 K_{01}+1\right] \text { and } M S E\left(\hat{R}_{(\alpha)}\right)=\theta R_{(\alpha)}^{2} A_{(\alpha)} .
$$

The $\operatorname{MSE}\left(\hat{R}_{(\alpha)}^{(h)}\right)$ and $\operatorname{MSE}\left(\hat{R}_{(\alpha)}^{(t)}\right)$ at $(1.9)$ and (1.10) are respectively minimized for

$$
h_{1}(1)=K_{(\alpha)} \text { and } t_{1}(1,1)=A, t_{2}(1,1)=B .
$$

Putting (1.12) in (1.9) and (1.10) yield the min.MSEs of $\hat{R}_{(\alpha)}^{(h)}$ and $\hat{R}_{(\alpha)}^{(t)}$ as

$$
\min \cdot \operatorname{MSE}\left(\hat{R}_{(\alpha)}^{(h)}\right)=\operatorname{MSE}\left(\hat{R}_{(\alpha)}\right)-\theta R_{(\alpha)}^{2} K_{(\alpha)}^{2} C_{2}^{2}
$$


and

$$
\min . M S E\left(\hat{R}_{(\alpha)}^{(t)}\right)=\operatorname{MSE}\left(\hat{R}_{(\alpha)}\right)-\theta R_{(\alpha)}^{2} B_{(\alpha)} .
$$

Thus we have established the following theorems:

Theorem 1.1 Up to terms of order $n^{-1}$,

$$
\operatorname{MSE}\left(\hat{R}_{(\alpha)}^{(h)}\right) \geq \operatorname{MSE}\left(\hat{R}_{(\alpha)}\right)-\theta R_{(\alpha)}^{2} K_{(\alpha)}^{2} C_{2}^{2}
$$

with equality holding if $h_{1}(1)=-K_{(\alpha)}$.

Theorem 1.2. Up to terms of order $n^{-1}$, with equality holding if $t_{1}(1,1)=A$ and $t_{2}(1,1)=B$.

Any parametric function $t(u, v)$ satisfying the regularity conditions can generate an asymptotically acceptable estimator. The following estimators:

$$
\begin{gathered}
d_{0(1)}=\hat{R}_{(\alpha)} u^{\alpha_{1}} v^{\alpha_{2}}, d_{0(2)}=\hat{R}_{(\alpha)}\left\{1+\alpha_{1}(u-1)\right\} /\left\{1+\alpha_{2}(v-1)\right\}, \\
d_{0(3)}=\hat{R}_{(\alpha)}\left[1-\alpha_{1}(u-1)-\alpha_{2}(v-1)\right]^{-1},
\end{gathered}
$$

$d_{0(4)}=\hat{R}_{(\alpha)}\left[1-\alpha_{1}(u-1)-a_{2}(v-1)\right], d_{0(5)}=\hat{R}_{(\alpha)}\left(2-u^{\alpha_{1}} v^{\alpha_{2}}\right)$, and $d_{0(6)}=$ $\hat{R}_{(\alpha)}\left[\alpha_{1} u+\left(1-\alpha_{1}\right) v^{\alpha_{2}}\right]$ etc. of the parameter $R_{(\alpha)}$ are members of the family of estimators $\hat{R}_{(\alpha)}^{(t)}$, where $\alpha_{1}$ and $\alpha_{2}$ are real constants. The optimum values of the constants $\alpha_{1}$ and $\alpha_{2}$ are obtained by the right hand sides of (1.12) and the resulting estimators will have the same minimum MSE given by (1.14). Further we note that the class of estimators $\hat{R}_{(\alpha)}^{(t)}$ does not include the simple differencetype estimator

$$
d_{0(7)}=\hat{R}_{(\alpha)}+\alpha_{1}(u-1)+\alpha_{2}(v-1) .
$$

This led authors to propose a class of estimators wider than $\hat{R}_{(\alpha)}^{(t)}$ in $(1.4)$, as

$$
\hat{R}_{(\alpha)}^{(T)}=T\left(\hat{R}_{(\alpha)}, u, v\right)
$$

where $T\left(\hat{R}_{(\alpha)}, u, v\right)$ is a function of $\left(\hat{R}_{(\alpha)}, u, v\right)$ such that $T(D)=R_{(\alpha)}, T_{1}(D)=$ 1 and satisfies certain regularity conditions as defined in the Appendix. To the first degree of approximation, the biases and MSEs of $\hat{R}_{(\alpha)}^{(H)}$ and $\hat{R}_{(\alpha)}^{(T)}$ are respectively given by

$$
B\left(\hat{R}_{(\alpha)}^{(H)}\right)=B\left(\hat{R}_{(\alpha)}\right)+\left(\frac{\theta}{2}\right) C_{2}^{2}\left[H_{22}\left(R_{(\alpha)}-1\right)+2 R_{(\alpha)} K_{(\alpha)} H_{12}\left(R_{(\alpha)}, 1\right)\right],
$$




$$
\begin{aligned}
& B\left(\hat{R}_{(\alpha)}^{(T)}\right)=B\left(\hat{R}_{(\alpha)}\right)+\left(\frac{\theta}{2}\right)\left[C_{2}^{2} T_{22}(D)+\left(\lambda_{004}-1\right) T_{33}(D)+2 R_{(\alpha)} K_{(\alpha)} T_{12}(D) C_{2}^{2}\right. \\
&\left.+2 R_{(\alpha)} d_{(\alpha)} T_{13}(D)+2 \lambda_{003} C_{2} T_{23}(D)\right] \\
& \operatorname{MSE}\left(\hat{R}_{(\alpha)}^{(H)}\right)=\operatorname{MSE}\left(\hat{R}_{(\alpha)}\right)+\theta C_{2}^{2} H_{2}\left(R_{(\alpha)}, 1,1\right)\left[H_{2}\left(R_{(\alpha)} 1,1\right)+2 R_{(\alpha)} K_{(\alpha)}\right]
\end{aligned}
$$

and

$$
\begin{aligned}
\operatorname{MSE}\left(\hat{R}_{(\alpha)}^{(T)}\right) & =\operatorname{MSE}\left(\hat{R}_{(\alpha)}\right)+\theta\left[C_{2}^{2} T_{2}^{2}(D)+\left(\lambda_{004}-1\right) T_{3}^{2}(D)\right. \\
& \left.+2 R_{(\alpha)} K_{(\alpha)} C_{2}^{2} T_{2}(D) 2 R_{(\alpha)} d_{(\alpha)} T_{3}(D)+\lambda_{003} C_{2} T_{2}(D) T_{3}(D)\right]
\end{aligned}
$$

The $\operatorname{MSE}\left(\hat{R}_{(\alpha)}^{(H)}\right)$ and $\operatorname{MSE}\left(\hat{R}_{(\alpha)}^{(T)}\right)$ in $(1.19)$ and $(1.20)$ respectively are minimized for

$$
H_{2}\left(R_{(\alpha)}, 1\right)=-R_{(\alpha)} K_{(\alpha)}
$$

and

$$
T_{2}(D)=R_{(\alpha)} A, T_{3}(D)=R_{(\alpha)} B
$$

Putting (1.21) and (1.22) respectively in (1.19) and (1.20), we get the minimum MSEs of $\hat{R}_{(\alpha)}^{(H)}$ and $\hat{R}_{(\alpha)}^{(T)}$ as

$$
\min \cdot \operatorname{MSE}\left(\hat{R}_{(\alpha)}^{(H)}\right)=\operatorname{MSE}\left(\hat{R}_{(\alpha)}\right)-\theta R_{(\alpha)}^{2} C_{2}^{2} K_{(\alpha)}^{2}=\min \cdot \operatorname{MSE}\left(\hat{R}_{(\alpha)}^{(h)}\right)
$$

and

$$
\min \cdot M S E\left(\hat{R}_{(\alpha)}^{(T)}\right)=\operatorname{MSE}\left(\hat{R}_{(\alpha)}\right)-\theta R_{(\alpha)}^{2} B_{(\alpha)}=\min \cdot M S E\left(\hat{R}_{(\alpha)}^{(t)}\right) .
$$

Thus it is proved that the asymptotic MSE for an optimum estimator of the family generated by $\hat{R}_{(\alpha)}^{(T)}\left(\hat{R}_{(\alpha)}^{(H)}\right)$ is same as the asymptotic MSE of an optimum estimator of the family $\hat{R}_{(\alpha)}^{(t)}\left(\hat{R}_{(\alpha)}^{(h)}\right)$ and is not further reduced.

From (1.11), (1.23) and (1.24), we have

$$
\operatorname{MSE}\left(\hat{R}_{(\alpha)}\right)-\left[\min \cdot \operatorname{MSE}\left(\hat{R}_{(\alpha)}^{(h)}\right)=\min \cdot M S E\left(\hat{R}_{(\alpha)}^{(H)}\right)\right]=\theta R_{(\alpha)}^{2} C_{2}^{2} K_{(\alpha)}^{2} \geq 0
$$

and

$$
\begin{aligned}
{\left[\min \cdot \operatorname{MSE}\left(\hat{R}_{(\alpha)}^{(h)}\right)\right.} & \left.=\min \cdot \operatorname{MSE}\left(\hat{R}_{(\alpha)}^{(H)}\right)\right] \\
& -\left[\min \cdot \operatorname{MSE}\left(\hat{R}_{(\alpha)}^{(t)}\right)=\min \cdot \operatorname{MSE}\left(\hat{R}_{(\alpha)}^{(T)}\right)\right] \\
& =\theta R_{(\alpha)}^{2} \frac{\Delta_{2}}{\Delta} \geq 0
\end{aligned}
$$


Thus from (1.25) and (1.26) we state the following theorem:

Theorem 1.3. The inequality

$$
\begin{gathered}
{\left[\min \cdot \operatorname{MSE}\left(\hat{R}_{(\alpha)}^{(t)}\right)=\min \cdot \operatorname{MSE}\left(\hat{R}_{(\alpha)}^{(T)}\right)\right]} \\
\leq\left[\min \cdot \operatorname{MSE}\left(\hat{R}_{(\alpha)}^{(h)}\right)=\min \cdot \operatorname{MSE}\left(\hat{R}_{(\alpha)}^{(H)}\right)\right] \leq \operatorname{MSE}\left(\hat{R}_{(\alpha)}\right)
\end{gathered}
$$

holds good at the optimum conditions.

It follows from Theorem 1.3 that the proposed family of estimators $\hat{R}_{(\alpha)}^{(t)}\left(\hat{R}_{(\alpha)}^{(T)}\right)$ is more efficient than the estimators $\hat{R}_{(\alpha)}, \hat{R}_{(\alpha)}^{(h)}$ and $\hat{R}_{(\alpha)}^{(H)}$. It is to be mentioned that $\hat{R}_{(\alpha)}^{(h)}$ is a member of the class $\hat{R}_{(\alpha)}^{(t)}$. The estimator $\hat{R}_{(\alpha)}^{(T)}$ includes all the estimators the $\hat{R}_{(\alpha)}, \hat{R}_{(\alpha)}^{(h)}, \hat{R}_{(\alpha)}^{(H)}$ and $\hat{R}_{(\alpha)}^{(t)}$. The biases and MSEs of the estimators $d_{0(i)}, i=1$ to 6 can be obtained from (1.8) and (1.10) just by putting the values of the derivatives as shown in scheme 1.1.

Scheme 1.1 Values of the derivatives.

\begin{tabular}{|c|c|c|c|c|c|}
\hline Estimator & $t_{1}(1.1)$ & $t_{2}(1,1)$ & $t_{11}(1,1)$ & $t_{12}(1,1)$ & $t_{22}(1,1)$ \\
\hline$d_{0(1)}$ & $\alpha_{1}$ & $\alpha_{2}$ & $\alpha_{1}\left(\alpha_{1}-1\right)$ & $\alpha_{1} \alpha_{2}$ & $\alpha_{2}\left(\alpha_{2}-1\right)$ \\
\hline$d_{0(2)}$ & $\alpha_{1}$ & $-\alpha_{2}$ & 0 & $-\alpha_{1} \alpha_{2}$ & $2 \alpha_{2}^{2}$ \\
\hline$d_{0(3)}$ & $\alpha_{1}$ & $\alpha_{2}$ & $2 \alpha_{1}^{2}$ & $2 \alpha_{1} \alpha_{2}$ & $2 \alpha_{2}^{2}$ \\
\hline$d_{0(4)}$ & $-\alpha_{1}$ & $-\alpha_{2}$ & 0 & 0 & 0 \\
\hline$d_{0(5)}$ & $-\alpha_{1}$ & $-\alpha_{2}$ & $-\alpha_{1}\left(\alpha_{1}-1\right)$ & $-\alpha_{1} \alpha_{2}$ & $-\alpha_{2}\left(\alpha_{2}-1\right)$ \\
\hline$d_{0(6)}$ & $\alpha_{1}$ & $\alpha_{2}\left(1-\alpha_{1}\right)$ & 0 & 0 & $\alpha_{2}\left(1-\alpha_{1}\right)\left(\alpha_{2}-1\right)$ \\
\hline
\end{tabular}

In this paper, we have studied the effect of random non-response on the family of estimators $\hat{R}_{(\alpha)}^{(h)}, \hat{R}_{(\alpha)}^{(H)}, \hat{R}_{(\alpha)}^{(t)}$ and $\hat{R}_{(\alpha)}^{(T)}$ in two situations advocated by Tracy and Osahan (1994): (i) non-response in the study as well as the auxiliary variables [Situation 1 ], and (ii) non-response in the study variable only [Situation 2]. The reader is referred to Singh and Joarder (1998), Singh and Singh (1979), Singh and Singh (1985), Singh, Joarder and Tracy (2000) and Singh and Tracy (2001).

\section{A Nonresponse Probability Model}

Let $\Omega:\left(v_{1}, v_{2}, \ldots, v_{N}\right)$ denote the population of $N$ units from which a simple random sample of size $n$ is drawn without replacement. If $r(r=0,1,2, \ldots,(n-2))$ denotes the number of sampling units on which information could not be obtained 
due to random non-response, then the remaining $(n-r)$ units in the sample can be treated as simple random sampling without replacement (SRSWOR) sample from $\Omega$. It is assumed that $r$ is less than $(n-1)$. We also assume that if $p$ denotes the probability of non-response among the $(n-2)$ possible values of non-response, then $r$ has the following discrete distribution as:

$$
P(r)=\frac{(n-r)}{n q+2 p} C_{r}^{n-2} q^{n-r-2}
$$

which is due to Singh and Joarder (1998), where $q=1-p$ and $r=0,1,2, \ldots,(n-$ 2). Let us define

$e_{0}=\frac{\bar{y}_{0(n-r)}}{\bar{Y}_{0}}-1, e_{1}=\frac{\bar{y}_{1(n-r)}}{\bar{Y}_{1}}, e_{2}=\frac{\bar{y}_{2(n-r)}}{\bar{Y}_{2}}-1, e_{3}=\frac{\bar{y}_{2}}{\bar{Y}_{2}}-1, e_{4} \frac{s_{2(n-r)}^{2}}{S_{2}^{2}}-1$,

and $e_{5}=\frac{s_{2}^{2}}{S_{2}^{2}}-1$.

incompleteness- in the form of missingness- is a trouble some feature of many data sets. Statisticians have identified for sometime that failure to account for the stochastic nature of incompleteness can damage the actual conclusion. An obvious problem arises what one needs to justify ignoring the incomplete mechanism. Rubin (1976) advocated three concepts: missing at random (MAR), observed at random (OAR), and parameter distribution (PD). Rubin defined, "The data are MAR if the probability of the observed missingness pattern, given the observed and unobserved data, does not depend on the value of the unobserved data". Heitjan and Basu (1996) have distinguished the meaning of missing at random (MAR) and missing completely at random (MCAR) in a very nice manner. The probability model defined at (2.1) is free from actual data values, hence can be considered as a model suitable for MAR situation. Then under the probability model given in (2.1), we have the following results:

$E\left(e_{i}\right)=0, i=0,1, \ldots 5$

$E\left(e_{0}^{2}\right)=\theta^{*} C_{0}^{2}, \quad E\left(e_{1}^{2}\right)=\theta^{*} C_{1}^{2}, \quad E\left(e_{2}^{2}\right)=\theta^{*} C_{2}^{2}, \quad E\left(e_{3}^{2}\right)=\theta C_{2}^{2}, \quad E\left(e_{4}^{2}\right)$

$=\theta^{*}\left(\lambda_{004}-1\right), \quad E\left(e_{5}^{2}\right)=\theta\left(\lambda_{040}-1\right), \quad E\left(e_{0} e_{1}\right)=\theta^{*} \rho_{01} C_{0} C_{1}, \quad E\left(e_{0} e_{2}\right)$

$=\theta^{*} \rho_{02} C_{0} C_{2}, \quad E\left(e_{0} e_{3}\right)=\theta \rho_{2} C_{0} C_{2}, \quad E\left(e_{0} e_{4}\right)=\theta^{*} \lambda_{102} C_{0}, \quad E\left(e_{0} e_{5}\right)=\theta \lambda_{102} C_{0}$,

$E\left(e_{1} e_{2}\right)=\theta^{*} \rho_{12} C_{1} C_{2}, \quad E\left(e_{1} e_{3}\right)=\theta \rho_{12} C_{1} C_{2}, \quad E\left(e_{1} e_{4}\right)=\theta^{*} \lambda_{012} C_{1}, \quad E\left(e_{1} e_{5}\right)$

$=\theta \lambda_{102} C_{1}, E\left(e_{2} e_{3}\right)=\theta C_{2}^{2}, \quad E\left(e_{2} e_{4}\right)=\theta^{*} \lambda_{003} C_{2}, \quad E\left(e_{2} e_{5}\right)=\theta \lambda_{003} C_{2}, \quad E\left(e_{3} e_{4}\right)$

$=\theta \lambda_{003} C_{2}, \quad E\left(e_{3} e_{5}\right)=\theta \lambda_{003} C_{2}$ and $E\left(e_{4} e_{5}\right)=\theta\left(\lambda_{004}-1\right)$.

It is to be noted that if $p=0$, that is, there is no non-response, the above expected values coincide with usual results. For practical examples of random non-response in survey sampling, the reader is referred to Tracy and Osahan (1994) and Toutenburg and Srivastava (1998). 


\section{$3 \quad$ Proposed Strategies}

In this section, we consider three different strategies depending upon the availability of information and non-response on both variables.

\subsection{Strategy I}

When random non-response for $r$ units on study variables $y_{0}, y_{1}$ and auxiliary variable $y_{2}$ is present in the sample, and population mean $\bar{Y}_{2}$ and variance $S_{2}^{2}$ are known, we define a family of estimators of $R_{(\alpha)}$ as:

$$
d_{1}=\hat{R}_{\alpha(n-r)} f\left(u_{(n-r)}, v_{(n-r)}\right)
$$

where $f\left(u_{(n-r)} v_{(n-r)}\right)$ is a function of $\left(u_{(n-r)}, v_{(n-r)}\right)$ such that $f(1,1)=1$ and it satisfies the following conditions:

(i) Whatever be the sample chosen, $\left(u_{(n-r)}, v_{(n-r)}\right)$ assumes values in a bounded, closed convex subset, $\mathbf{S}$, of the two-dimensional real space containing the point $(1,1)$.

(ii) In $\mathbf{S}$, the function $f\left(u_{(n-r)}, v_{(n-r)}\right)$ is continuous and bounded.

(iii) The first and second order partial derivatives of $f\left(u_{(n-r)}, v_{(n-r)}\right)$ exist and are continuous and bounded in $\mathbf{S}$.

The bias and MSE of $d_{1}$ to the first degree of approximation, are respectively given by

$$
\begin{aligned}
B\left(d_{1}\right)=B\left(\hat{R}_{\alpha(n-r)}\right) & +\left(\frac{\theta^{*} R_{(\alpha)}}{2}\right)\left[2 K_{(\alpha)} C_{2}^{2} f_{1}(1,1)+2 d_{(\alpha)} f_{2}(1,1)+C_{2}^{2} f_{11}(1,1)\right. \\
& \left.+2 \lambda_{003} C_{2} f_{12}(1,1)+\left(\lambda_{004}-1\right) f_{22}(1,1)\right]
\end{aligned}
$$

and

$$
\begin{gathered}
M S E\left(d_{1}\right)=\operatorname{MSE}\left(\hat{R}_{\alpha(n-r)}\right)+\theta^{*} R_{(\alpha)}^{2}\left[C_{2}^{2} f_{1}^{2}(1,1)+\left(\lambda_{004}-1\right) f_{2}^{2}(1,1)\right. \\
\left.+2 \lambda_{003} C_{2} f_{1}(1,1) f_{2}(1,1)+2 K_{(\alpha)} C_{2}^{2} f_{1}(1,1)+2 d_{(\alpha)} f_{2}(1,1)\right]
\end{gathered}
$$

where

$$
B\left(\hat{R}_{\alpha(n-r)}\right)=\theta^{*} R_{(\alpha)}\left(\frac{\alpha C_{1}^{2}}{2}\right)\left(\alpha-2 K_{01}+1\right)
$$

and

$$
\operatorname{MSE}\left(\hat{R}_{\alpha(n-r)}\right)=\theta^{*} R_{(\alpha)}^{2}\left[C_{0}^{2}+\alpha C_{1}^{2}\left(\alpha-2 K_{01}\right)\right]
$$


are the bias and MSE of $\hat{R}_{\alpha(n-r)}$ to the first degree of approximation, respectively. The optimum values of $f_{1}(1,1)$ and $f_{2}(1,1)$ for which $\operatorname{MSE}\left(d_{1}\right)$ is minimum, are given by

$$
f_{1}(1,1)=A \text { and } f_{2}(1,1)=B
$$

Substitution of (3.6) in (3.3) yields the minimum MSE of $d_{1}$ as

$$
\min \cdot M S E\left(d_{1}\right)=M S E\left(\hat{R}_{\alpha(n-r)}\right)-\theta^{*} R_{(\alpha)}^{2} B_{(\alpha)} .
$$

Thus we state the following theorem.

Theorem 3.1. Up to terms of order $n^{-1}$

$$
\left.\operatorname{MSE}\left(d_{1}\right) \geq \operatorname{MSE}\left(\hat{R}_{\alpha(n-r}\right)\right)-\theta^{*} R_{(\alpha)}^{2} B_{(\alpha)}
$$

with equality holding if $f_{1}(1,1)=A$ and $f_{2}(1,1)=B$.

A family of estimators wider than $d_{1}$ is defined by

$$
d_{2}=F\left(\hat{R}_{\alpha(n-r)}, u_{(n-r)}, v_{(n-r)}\right)
$$

where the function $F\left(\hat{R}_{\alpha(n-r)}, u_{(n-r)}, v_{(n-r)}\right)$ satisfies $F(D)=R_{(\alpha)}$ and $F_{1}(D)=1$.

To the first degree of approximation the bias and MSE of $d_{2}$ are respectively given by

$$
\begin{aligned}
B\left(d_{2}\right)= & B\left(\hat{R}_{\alpha(n-r)}\right)+\left(\frac{\theta^{*}}{2}\right)\left[C_{2}^{2} F_{22}(D)+\left(\lambda_{004}-1\right) F_{33}(D)\right. \\
& \left.+2 R_{(\alpha)} K_{(\alpha)} F_{12}(D) 2 R_{(\alpha)} d_{\alpha)} F_{13}(D)+2 \lambda_{003} C_{2} F_{23} D\right]
\end{aligned}
$$

and

$$
\begin{aligned}
M S E\left(d_{2}\right)= & M S E\left(\hat{R}_{\alpha(n-r)}\right)+\theta^{*}\left[C_{2}^{2} F_{2}^{2}(D)+\left(\lambda_{004}-1\right) F_{3}^{2}(D)+2 R_{(\alpha)} K_{(\alpha)} C_{2}^{2}\right. \\
& \left.F_{12}(D)+2 R_{(\alpha)} d_{(\alpha)} F_{13}(D)+2 \lambda_{003} C_{2} F_{23}(D)\right] .
\end{aligned}
$$

The $\operatorname{MSE}\left(d_{2}\right)$ is minimized for

$$
F_{2}(D)=R_{(\alpha)} A=A^{*} \text { (say) and } F_{3}(D)=R_{(\alpha)} B=B^{*} \text { (say) }
$$

Thus the minimum MSE of $d_{2}$ is given by

$$
\operatorname{MSE}\left(d_{2}\right)=\operatorname{MSE}\left(\hat{R}_{\alpha(n-r)}\right)-\theta^{*} R_{(\alpha)}^{2} B_{(\alpha)}=\min \cdot \operatorname{MSE}\left(d_{1}\right)
$$

It follows from (3.12) that to the first degree of approximation, the minimum MSE of $d_{2}$ is equal to that of $d_{1}$ (i.e. $\left.\min \cdot \operatorname{MSE}\left(d_{2}\right)=\min \cdot \operatorname{MSE}\left(d_{1}\right)\right)$ and is not reduced. 
Further we note that the estimators of the type

$$
d_{2(1)}=\hat{R}_{\alpha(n-r)}+\alpha_{1}\left(u_{(n-r)}-1\right), d_{2(2)}=\frac{\bar{y}_{0(n-r)}+\alpha_{1}\left(u_{(n-r)}-1\right)}{\bar{y}_{1(n-r)}+\alpha_{2}\left(u_{(n-r)}-1\right)}
$$

are members of the family $d_{2}$ but not of $d_{1}$, where $\alpha_{i}, i=1,2$ are real constants.

Remark 3.1. The following families of estimators of $R_{(\alpha)}$ :

$$
d_{1(1)}=\hat{R}_{\alpha(n-r)} f\left(u_{(n-r)}\right), \quad\left(\text { only } \bar{Y}_{2} \text { of } y_{2}\right. \text { is known) }
$$

and

$$
d_{1(2)}=\hat{R}_{\alpha(n-r)} f\left(v_{(n-r)}\right), \quad\left(\text { only } S_{2}^{2} \text { of } y_{2}\right. \text { is known) }
$$

are members of the family $d_{1}$, where $f\left(u_{(n-r)}\right)$ and $f\left(v_{(n-r)}\right.$ are the functions of $u_{(n-r)}$ and $v_{(n-r)}$ respectively such that $f(1)=1$. The bias of $d_{1(1)}$ and $d_{1(2)}$ can be obtained from (3.2) just by putting $\left[f_{1}(1,1)=f_{1}(1), \quad f_{11}(1,1)=f_{11}(1), \quad f_{2}(1,1)\right.$ $\left.=f_{12}(1,1)=f_{22}(1,1)=0\right]$ and $\left[f_{2}(1,1)=f_{2}(1), \quad f_{22}(1,1)=f_{22}(1)\right.$, and $f_{1}(1,1)$ $\left.=f_{12}(1,1)=f_{11}(1,1)=0\right]$. The MSEs of $d_{1(1)}$ and $d_{1(2)}$ can be obtained from (3.3) by putting $\left[f_{1}(1,1)=f_{1}(1), \quad f_{2}(1)=1\right]$ and $\left[f_{2}(1,1)=f_{2}(1), f_{1}(1)=1\right]$. The MSEs of $d_{1(1)}$ and $d_{1(2)}$ are respectively minimied for

$$
f_{1}(1)=-K_{(\alpha)}
$$

and

$$
f_{2}(1)=-d_{(\alpha)} /\left(\lambda_{004}-1\right)
$$

which give the minimum MSEs of $d_{1(1)}$ and $d_{1(2)}$ respectively as

$$
\min \cdot \operatorname{MSE}\left(d_{1(1)}\right)=\operatorname{MSE}\left(\hat{R}_{\alpha(n-r)}\right)-\theta^{*} R_{(\alpha)}^{2} C_{2}^{2} K_{(\alpha)}^{2}
$$

and

$$
\min \cdot \operatorname{MSE}\left(d_{1(2)}\right)=\operatorname{MSE}\left(\hat{R}_{\alpha(n-r)}\right)-\theta^{*} R_{(\alpha)}^{2} d_{(\alpha)}^{2} /\left(\lambda_{004}-1\right)
$$

Remark 3.2. The following families of estimators of $R_{(\alpha)}$ :

$$
d_{2(3)}=F\left(\hat{R}_{\alpha(n-r)}, u_{(n-r)}\right),\left(\text { only } \bar{Y}_{2} \text { of } y_{2} \text { is known }\right)
$$

and

$$
d_{2(4)}=F\left(\hat{R}_{\alpha(n-r)}, v_{(n-r)}\right),\left(\text { only } S_{2}^{2} \text { of } y_{2} \text { is known }\right)
$$

where $F\left(\hat{R}_{\alpha(n-r)}, u_{(n-r)}\right)$, and $F\left(\hat{R}_{\alpha(n-r)}, v_{(n-r)}\right)$ are the functions of $\left(\hat{R}_{\alpha(n-r)}, u_{(n-r)}\right)$ and $\left(\hat{R}_{\alpha(n-r)}, v_{(n-r)}\right)$ such that $F\left(R_{\alpha} 1\right)=R_{(\alpha)}$ and $F_{1}\left(R_{(\alpha)}, 1\right)=1$. 
The biass of $d_{2(3)}$ and $d_{2(4)}$ can be obtained from (3.9) by putting $\left[F_{22}(D)=F_{22}\left(R_{(\alpha)}, 1\right), F_{12}(D)=F_{12}\left(R_{(\alpha)}, 1\right), F_{13}(D)=F_{23}(D)=F_{33}(D)=0\right]$ and $\left[F_{33}(D)=F_{33}\left(R_{(\alpha)}, 1\right), F_{13}(D)=F_{13}\left(R_{(\alpha)}, 1\right), F_{22}(D)=F_{12}(D)=F_{23}(D)=0\right]$ respectively. The MSEs of $d_{2(3)}$ and $d_{2(4)}$ can be obtained from (3.10) by putting $\left[F_{2}(D)=F_{2}\left(R_{(\alpha)}, 1\right) F_{3}(D)=0\right]$ and $\left[F_{3}(D)=F_{3}\left(R_{(\alpha)}, 1\right), F_{2}(D)=0\right]$ respectively. The MSEs of $d_{2(3)}$ and $d_{2(4)}$ are respectively minimized for

$$
F_{2}\left(R_{(\alpha)}, 1\right)=-R_{(\alpha)} K_{(\alpha)}
$$

and

$$
F_{3}\left(R_{\alpha)}, 1\right)=-R_{(\alpha)} d_{\alpha} /\left(\lambda_{004}-1\right)
$$

Thus the resulting minimum MSEs of $d_{2(3)}$ and $d_{2(4)}$ are respectively given by

$$
\min \cdot \operatorname{MSE}\left(d_{1(1)}\right)=\min \cdot \operatorname{MSE}\left(d_{2(3)}\right)=\operatorname{MSE}\left(\hat{R}_{\alpha(n-r)}\right)-\theta^{*} R_{(\alpha)}^{2} C_{2}^{2} K_{(\alpha)}^{2}
$$

and

$\min \cdot \operatorname{MSE}\left(d_{1(2)}\right)=\min \cdot \operatorname{MSE}\left(d_{2(4)}\right)=\operatorname{MSE}\left(\hat{R}_{\alpha(n-r)}\right)-\theta^{*} R_{(\alpha)}^{2} d_{(\alpha)}^{2} d_{(\alpha)}^{2} /\left(\lambda_{004}-1\right)$

Now we state the following corollary:

Corollary 3.1. Up to terms of order $n^{-1}$,

(i) $\operatorname{MSE}\left(d_{1(1)}\right.$ or $\left.d_{2(3)}\right) \geq\left[\operatorname{MSE}\left(\hat{R}_{\alpha(n-r)}\right)-\theta^{*} R_{(\alpha)}^{2} C_{2}^{2} K_{(\alpha)}^{2}\right]$ with equality holding if $f_{1}(1)=-K_{(\alpha)}$ for $d_{1(1)}$, and if $F_{2}\left(R_{(\alpha)}, 1\right)$ $=-R_{(\alpha)} K_{(\alpha)}$ for $d_{2(3)}$.

(ii) $\operatorname{MSE}\left(d_{1(2)}\right.$ or $\left.d_{2(4)}\right) \geq\left\lfloor\operatorname{MSE}\left(\hat{R}_{\alpha(n-r)}\right)-\theta^{*} R_{(\alpha)}^{2} d_{(\alpha)}^{2} /\left(\lambda_{004}-1\right)\right\rfloor$ with equality holding if $f_{2}(1)=d_{(\alpha)} /\left(\lambda_{004}-1\right)$, and if $F_{3}\left(R_{(\alpha)}, 1\right)$ $=-R_{(\alpha)} d_{(\alpha)} /\left(\lambda_{004}-1\right)$ for $d_{2(4)}$.

Remark 3.3. It is to be mentioned here that all the families of estimators $d_{1}, d_{1(1)}, d_{1(2)}, d_{2(3)}$ and $d_{2(4)}$ are the members of the family $d_{2}$. The biases and MSEs of these estimators can be obtained from (3.9) and (3.10) respectively by putting the suitable values of the derivatives as per scheme 3.1: 
Scheme 3.1 Values of the derivatives

\begin{tabular}{|c|c|c|c|c|}
\hline Estimator & $F_{2}(D)$ & $F_{3}(D)$ & $F_{12}(D)$ & $F_{13}(D)$ \\
\hline$d_{1}$ & $R_{(\alpha)} f_{1}(1,1)$ & $R_{(\alpha)} f_{2}(1,1)$ & $f_{1}(1,1)$ & $f_{2}(1,1)$ \\
\hline$d_{1(1)}$ & $R_{(\alpha)} f_{1}(1)$ & 0 & $f_{1}(1)$ & 0 \\
\hline$d_{1(2)}$ & 0 & $R_{(\alpha)} f_{2}(1)$ & 0 & $f_{2}(1)$ \\
\hline$d_{2(3)}$ & $F_{2}\left(R_{(\alpha)},(1)\right.$ & 0 & $f_{12}\left(R_{(\alpha)},(1)\right.$ & 0 \\
\hline$d_{2(4)}$ & 0 & $F_{3}\left(R_{(\alpha)}, 1\right)$ & 0 & $F_{13}\left(R_{(\alpha)},(1)\right.$ \\
\hline
\end{tabular}

\begin{tabular}{|c|c|c|c|}
\hline Estimator & $F_{22}(D)$ & $F_{23}(D)$ & $F_{33}(D)$ \\
\hline$d_{1}$ & $R_{(\alpha)} f_{11}(1,1)$ & $R_{(\alpha)} f_{12}(1,1)$ & $R_{(\alpha)} f_{22}(1,1)$ \\
\hline$d_{1(1)}$ & $R_{(\alpha)} f_{11}(1)$ & 0 & 0 \\
\hline$d_{1(2)}$ & 0 & 0 & $R_{(\alpha)} f_{22}(1)$ \\
\hline$d_{2(3)}$ & $F_{22}\left(R_{(\alpha)}, 1\right)$ & 0 & 0 \\
\hline$d_{2(4)}$ & 0 & 0 & $F_{33}\left(R_{(\alpha)}, 1\right)$ \\
\hline
\end{tabular}

Remark 3.4. If we set $\alpha=0$ in (3.1), (3.8), we get the families of estimators of population mean $\bar{Y}_{0}$ as

$$
d_{1}^{(1)}=\bar{y}_{0(n-r)} f\left(u_{(n-r)}, v_{(n-r)}\right)
$$

and

$$
d_{2}^{(1)}=F\left(\bar{y}_{0(n-r)}, u_{(n-r)}, v_{(n-r)}\right)
$$

where $f\left(u_{(n-r)}, v_{(n-r)}\right)$ and $F\left(\bar{y}_{0(n-r)}, u_{(n-r)}, v_{(n-r)}\right)$ are the functions of $\left(u_{(n-r)}, v_{(n-r)}\right)$ and $\left(\bar{y}_{0(n-r)}, u_{(n-r)}, v_{(n-r)}\right)$ respectively such that $f(1,1)=1$ and $F\left(D_{0}\right)=\bar{Y}_{0}$ implies that $F_{1}\left(D_{0}\right)=1$. The biases and MSEs of $d_{1}^{(1)}$ and $d_{2}^{(1)}$ can be obtained from $((3.2,(3.3))$ and $((3.9),(3.10))$ respectively by putting $\alpha=0$. The optimum values of $\left(f_{1}(1,1), f_{2}(1,1)\right)$ and $\left.F_{2}\left(D_{0}\right), F_{3}\left(D_{0}\right)\right)$ for which the MSEs of $d_{1}^{(1)}$ and $d_{2}^{(1)}$ are minimum, and the minimum MSEs of $d_{1}^{(1)}$ and $d_{2}^{(1)}$ can be obtained from $((3.6),(3.11))$ and $((3.7,(3.12))$ respectively by putting $\alpha=0$. The common min.MSE of $d_{1}^{(1)}$ and $d_{2}^{(1)}$ is

$$
\min \cdot \operatorname{MSE}\left(d_{1}^{(1)}\right)=\min \cdot \operatorname{MSE}\left(d_{2}^{(1)}\right)=\theta^{*} S_{0}^{2}\left[1-\rho_{02}^{2}-\left(\lambda_{003} \rho_{02}-\lambda_{102}\right)^{2} / \triangle\right] .
$$

Thus putting $\alpha=0$ in the theorem 3.1, we get the following corollaries:

Corollary 3.2. Upto terms of order $n^{-1}$,

(i) $\operatorname{MSE}\left(d_{1}^{(1)}\right) \geq \theta^{*} S_{0}^{2}\left[1-\rho_{02}^{2}-\left(\lambda_{003 \rho_{02}-\lambda_{102}}\right)^{2} / \triangle\right]$ with equality holding if $f_{1}(1,1)=A_{11}$ and $f_{2}(1,1)=B_{11}$,

(ii) $\operatorname{MSE}\left(d_{2}^{(1)}\right) \geq \theta^{*} S_{0}^{2}\left[1-\rho_{02}^{2}-\left(\lambda_{003} \rho_{02}-\lambda_{102}\right)^{2} / \triangle\right]$ 
with equality holding if $F_{2}\left(D_{0}\right)=\bar{Y}_{0} A_{11}$ and $F_{3}\left(D_{0}\right)=\bar{Y}_{0} B_{11}$, where $A_{11}$ and $B_{11}$ can be derived from $A$ and $B$ respectively by putting $\alpha=0$.

Remark 3.5. The following classes of estimators of population mean $\bar{Y}_{0}$ :

$$
\begin{aligned}
& d_{1(1)}^{1}=\bar{y}_{0(n-r)} f\left(u_{(n-r)}\right),\left(\text { only } \bar{Y}_{2} \text { of } y_{2}\right. \text { is known), } \\
& d_{1(2)}^{1}=\bar{y}_{0(n-r)} f\left(v_{(n-r)}\right),\left(\text { only } S_{2}^{2} \text { of } y_{2} \text { is known }\right), \\
& d_{2(1)}^{1}=F\left(\bar{y}_{0(n-r)}, u_{(n-r)}\right),\left(\text { only } \bar{Y}_{2} \text { of } y_{2}\right. \text { is known), } \\
& d_{2(2)}^{1}=F\left(\bar{y}_{0(n-r)}, v_{n-r)}\right),\left(\text { only } S_{2}^{2} \text { of } y_{2}\right. \text { is known), }
\end{aligned}
$$

where $f\left(u_{(n-r)}\right), f\left(v_{(n-r)}\right), F\left(\bar{y}_{0(n-r)}, u_{(n-r)}\right)$ and $F\left(\bar{y}_{0(n-r)}, v_{(n-r)}\right)$ are the functions of $u_{(n-r)}, v_{(n-r)}$, and $\left(\bar{y}_{0(n-r)}, u_{(n-r)}\right)$ and $\left(\bar{y}_{0(n-r)}, v_{(n-r)}\right)$ respectively such that $f(1)=1$ and $F\left(\bar{Y}_{0}, 1\right)=\bar{Y}_{0}$ which implies that $F_{1}\left(\bar{Y}_{0}, 1\right)=1$. It is to be noted that (i) $d_{1(1)}^{1}$ and $d_{1(2)}^{1}$, and (ii) $d_{2(1)}^{1}$ and $d_{2(2)}^{1}$, are the members of $d_{1}$ and $d_{2}$ respectively. Thus the biases and MSEs of these estimators can be obained from (3.9) and (3.10) respectively by putting $\alpha=0$ and the suitable values of the derivations from in scheme 3.2 .

Scheme 3.2 Values of the derivatives.

\begin{tabular}{|c|c|c|c|c|}
\hline Estimator & $F_{2}(D)$ & $F_{3}(D)$ & $F_{12}(D)$ & $F_{13}(D)$ \\
\hline$d_{1}^{(1)}$ & $\bar{Y}_{0} f_{1}(1,1)$ & $\bar{Y}_{0} f_{2}(1,1)$ & $f_{1}(1,1)$ & $f_{2}(1,1)$ \\
\hline$d_{2}^{(1)}$ & $F_{2}\left(D_{0}\right)$ & $F_{3}\left(D_{0}\right)$ & $F_{12}\left(D_{0}\right)$ & $F_{13}\left(D_{0}\right)$ \\
\hline$d_{1(1)}^{(1)}$ & $\bar{Y}_{0} f_{1}(1)$ & 0 & $f_{1}(1)$ & 0 \\
\hline$d_{1(2)}^{(1)}$ & 0 & $\bar{Y}_{0} f_{2}(1)$ & 0 & $f_{2}(1)$ \\
\hline$d_{2(1)}^{(1)}$ & $F_{2}\left(\bar{Y}_{0}, 1\right)$ & 0 & $F_{12}\left(\bar{Y}_{0}, 1\right)$ & 0 \\
\hline$d_{2(2)}^{(1)}$ & 0 & $F_{3}\left(\bar{Y}_{0}, 1\right)$ & 0 & $F_{13}\left(\bar{Y}_{0}, 1\right)$ \\
\hline
\end{tabular}

\begin{tabular}{|c|c|c|c|}
\hline Estimator & $F_{22}(D)$ & $F_{23}(D)$ & $F_{33}(D)$ \\
\hline$d_{1}^{(1)}$ & $\bar{Y}_{0} f_{11}(1,1)$ & $\bar{Y}_{0} f_{12}(1,1)$ & $\bar{Y}_{0} f_{22}(1,1)$ \\
\hline$d_{2}^{(1)}$ & $F_{22}\left(D_{0}\right)$ & $F_{23}\left(D_{0}\right)$ & $F_{33}\left(D_{0}\right)$ \\
\hline$d_{1(1)}^{(1)}$ & $\bar{Y}_{0} f_{11}(1)$ & 0 & 0 \\
\hline$d_{1(2)}^{(1)}$ & 0 & 0 & $\bar{Y}_{0} f_{22}(1)$ \\
\hline$d_{2(1)}^{(1)}$ & $F_{22}\left(\bar{Y}_{0}, 1\right)$ & 0 & 0 \\
\hline$d_{2(2)}^{(1)}$ & 0 & 0 & $F_{33}\left(\bar{Y}_{0}, 1\right)$ \\
\hline
\end{tabular}

The optimum values of the derivatives for which the MSEs of $d_{1(1)}^{(1)}, d_{1(2)}^{(1)}, d_{2(1)}^{(1)}$, and $d_{2(2)}^{(1)}$ are minimum and the minimum MSEs of these estimators can be obtained from 
(3.15), (3.16), (3.17), (3.18), (3.21), (3.22), (3.23), and (3.24) just by putting $\alpha=0$. The minimum MSEs of $d_{1(1)}^{(1)}, d_{1(2)}^{(1)}, d_{2(1)}^{(1)}$, and $d_{2(2)}^{(1)}$ are respectively given by

$$
\min \cdot \operatorname{MSE}\left(d_{1(1)}^{(1)}\right)=\min \cdot \operatorname{MSE}\left(d_{2(1)}^{(1)}\right)=\theta^{*} S_{0}^{2}\left(1-\rho_{02}^{2}\right)
$$

and

$$
\min \cdot \operatorname{MSE}\left(d_{1(2)}^{(1)}\right)=\min \cdot \operatorname{MSE}\left(d_{2(2)}^{(1)}\right)=\theta^{*} S_{0}^{2}\left(1-\lambda_{102}^{2} /\left(\lambda_{004}-1\right)\right) .
$$

Thus we state the following corollary by putting $\alpha=0$ in corollary 3.1.

Corollary 3.3. Up to terms of order $n^{-1}$

(i) $\operatorname{MSE}\left(d_{1(1)}^{(1)}\right.$ or $\left.d_{2(1)}^{(1)}\right) \geq \theta^{*} S_{0}^{2}\left(1-\rho_{02}^{2}\right)$ with equality holding if $f_{1}(1)=-K_{02}$ for $d_{1(1)}^{(1)}$, and if $F_{2}\left(\bar{Y}_{0}, 1\right)=-\bar{Y}_{0} K_{02}$ for $d_{2(1)}^{(1)}$.

(ii) $\operatorname{MSE}\left(d_{1(2)}^{(1)}\right.$ or $\left.d_{2(2)}^{(1)}\right) \geq \theta^{*} S_{0}^{2}\left(1-\lambda_{102}^{2} /\left(\lambda_{004}-1\right)\right)$

with equality holding if $f_{2}(1)=-\lambda_{102} C_{0} /\left(\lambda_{004}-1\right)$ for $d_{1(2)}^{(1)}$,

and if $F_{3}\left(\bar{Y}_{0}, 1\right)=-\bar{Y}_{0} \lambda_{102} C_{0} /\left(\lambda_{004}-1\right)$ for $d_{2(2)}^{(1)}$.

It is to be noted that the estimator

$$
\left.d_{2(3)}^{(1)}=\bar{y}_{0(n-r)}-\alpha_{1} \bar{Y}_{2}\left(u_{(n-r)}\right)-\right)
$$

suggested by Singh, Joarder and Tracy (2000) is a particular member of the family $d_{2(1)}^{(1)}$ but not of $d_{1(1)}^{(1)}$, where $\alpha_{1}$ is a constant. The minimum MSE of $d_{2(3)}^{(1)}$ is the same as given by (3.32).

\subsubsection{Estimators with Estimated Optimum Values}

It is to be noted that the optimum values of the parameters involved in estimators depend on unknown population parameters such as $\lambda_{003}, \lambda_{004}, \lambda_{102}, \lambda_{012}, C_{2}$, $K_{01}, K_{02}, \ldots$ etc. to use such an estimator one has to use guessed values or estimated values of these parameters. Guessed values of population parameters can be obtained from either past data or experience. If the guessed values are not known then it is advisable to use sample data at hand to estimate these parameters. The estimated optimum values of $f_{1}(1,1)$ and $f_{2}(1,1)$ are given by

$$
\hat{f}_{1}(1,1)=\hat{A} \quad \text { and } \quad \hat{f}_{2}(1,1)=\hat{B} .
$$


In case of estimated optimum values we find the MSE as: From the regularity conditions, defined in the Appendix, for $d_{1}$ we desire a function $f\left(u_{(n-r)}, v_{(n-r)}\right)$ such that $f(1,1)=1, f_{1}(1,1)=A, f_{2}(1,1)=B$, which indicates that the function $f\left(u_{(n-r)}, v_{(n-r)}\right)$ will contain not only $u_{(n-r)}, v_{(n-r)}$ but $A$ and $B$ as well, and thus we need a function $f^{*}\left(u_{(n-r)}, v_{(n-r)}, A, B\right)$ such that $f^{*}(Z)=$ $1, f_{1}^{*}(Z)=A, f_{2}^{*}(Z)=B$. Since in such function $f^{*}\left(u_{(n-r)}, v_{(n-r)}, A, B\right)$ so required, $A$ and $B$ are unknown, thus we may take $f^{* *}\left(u_{(n-r)}, v_{(n-r)}, \hat{A}, \hat{B}\right)=$ $f^{*}\left(u_{(n-r)}, v_{(n-r)}, \hat{A}, \hat{B}\right)$ (replacing $A, B$ by their estimated values). Now $f^{*}(Z)=$ $1, f_{1}^{*}(Z)=A, f_{2}^{*}(Z)=B$.

We may consider

$$
d_{1}^{*}=\hat{R}_{\alpha(n-r)} f^{*}\left(u_{(n-r)}, v_{(n-r)}, \hat{A}, \hat{B}\right)
$$

as an estimator of $R_{(\alpha)}$. Expanding $f^{*}\left(u_{(n-r)}, v_{(n-r)}, \hat{A}, \hat{B}\right)$ about the point $Z$ in Taylor's series, we have

$$
\begin{aligned}
d_{1}^{*} & =\hat{R}_{\alpha(n-r)}\left[1+\left(u_{(n-r)-1}\right) f_{1}^{*}(Z)+\left(v_{(n-r)}-1\right) f_{2}^{*}(Z)\right. \\
& \left.+(\hat{A}-A) f_{3}^{*}(Z)+(\hat{B}-B) f_{4}^{*}(Z)+\ldots\right]
\end{aligned}
$$

Then in terms of $e_{i}$ 's, we have

$$
\begin{aligned}
& d_{1}^{*}-\hat{R}_{(\alpha)}=R_{\alpha}\left[e_{0}-\alpha e_{1}+e_{2} A+e_{4} B+\left(e_{0}-\alpha e_{1}\right) e_{2} A+\left(e_{0}-\alpha e_{1}\right) e_{4} B\right. \\
& \left.\quad+(\hat{A}-A) f_{3}^{*}(Z)+(\hat{B}-B) f_{4}^{*}(Z)+\ldots\right] .
\end{aligned}
$$

Squaring both sides of (3.36) and taking expectation, we have the MSE of $d_{1}^{*}$ be equal to the minimum MSE of $d_{1}$ in $(3.7)$ if $f_{3}^{*}(Z)=0, f_{4}^{*}(Z)=0$. Thus if we consider the family of estimators (depending upon estimated optimum values)

$$
d_{1}^{*}=\hat{R}_{\alpha(n-r)} f^{*}\left(u_{(n-r)}, v_{(n-r)}, \hat{A}, \hat{B}\right)
$$

such that $f^{*}(Z)=1, f_{1}^{*}(Z)=A, f_{2}^{*}(Z)=B, f_{3}^{*}(Z)=0$ and $f_{4}^{*}(Z)=0$ such an estimator $d_{1}^{*}$ attains the $\min \cdot \operatorname{MSE}\left(d_{1}\right)$ given in (3.7).

Now we state the following theorems, and proofs can also be obtained by following Randles (1982).

Theorem 3.2. The family of estimators (based on estimated optimum values) of $R_{(\alpha)}$ defined by

$$
d_{1}^{*}=\hat{R}_{\alpha(n-r)} f^{*}\left(u_{(n-r)}, v_{(n-r)}, \hat{A}, \hat{B}\right)
$$


has the MSE, to the first degree of approximation, equal to the minimum MSE of $d_{1}$ that is

$$
\operatorname{MSE}\left(d_{1}^{*}\right)=\operatorname{MSE}\left(\hat{R}_{\alpha(n-r)}\right)-\theta^{*} R_{(\alpha)}^{2} B_{(\alpha)}=\min \cdot \operatorname{MSE}\left(d_{1}\right)
$$

where $f^{*}\left(u_{(n-r)}, v_{(n-r)}, \hat{A}, \hat{B}\right)$ is a function of $\left(u_{(n-r)}, v_{(n-r)}, \hat{A}, \hat{B}\right)$ such that $f^{*}(Z)=1, \quad f_{1}^{*}(Z)=A, \quad f_{2}^{*}(Z)=B, \quad f_{3}^{*}(Z)=0$, and $f_{4}^{*}(Z)=0$.

Theorem 3.3. A wider family of estimators (based on estimated optimum values) of $R_{(\alpha)}$ defined by $d_{2}^{*}=F^{*}\left(\hat{R}_{\alpha(n-r)}, u_{(n-r)}, v_{(n-r)}, \hat{A}, \hat{B}\right)$ has the MSE, to the first degree of approximation, same as the min.MSE of $d_{2}$ given by (3.12) i.e.

$$
\operatorname{MSE}\left(d_{2}^{*}\right)=\operatorname{MSE}\left(\hat{R}_{\alpha(n-r)}\right)-\theta^{*} R_{(\alpha)}^{2} B_{(\alpha)}=\min \cdot \operatorname{MSE}\left(d_{2}\right)
$$

where $F\left(\hat{R}_{\alpha(n-r)}, u_{(n-r)}, v_{(n-r)}, \hat{A}, \hat{B}\right)$ is a function of $\left(\hat{R}_{\alpha(n-r)}, u_{(n-r)}, v_{(n-r)}, \hat{A}, \hat{B}\right)$ such that $F^{*}\left(Q^{*}\right)=R_{(\alpha)}, F_{1}^{*}\left(Q^{*}\right)=1, F_{2}^{*}\left(Q^{*}\right)=A^{*}, F_{3}^{*}\left(Q^{*}\right)=B^{*}, F_{4}^{*}\left(Q^{*}\right)=$ 0 and $F_{5}^{*}\left(Q^{*}\right)=0$.

In similar fashion many other families of estimators of ratio $R_{(\alpha)}$ based on estimated optimum values can be derived. Further we state the following theorem:

Theorem 3.4. Estimators for estimating the minimum MSE of $d_{1}\left(\right.$ or $d_{2}$ ) or MSE of $d_{1}^{*}\left(\right.$ or $\left.\left.d_{2}^{*}\right)\right), d_{1}^{(1)}\left(\right.$ or $\left.d_{2}^{(1)}\right)$, and $d_{1(1)}^{(1)}\left(\right.$ or $\left.d_{2(1)}^{(1)}\right)$ are respectively given by

$$
\begin{aligned}
\min . \operatorname{MSE}\left(d_{1}\right)= & \min \cdot \operatorname{MSE}\left(d_{2}\right)=\operatorname{MS} \mathrm{S}\left(d_{2}^{*}\right)=\operatorname{MSE}\left(\hat{R}_{\alpha(n-r)}\right) \\
- & \hat{\theta}^{* 2} \hat{R}_{\alpha(n-r)}^{2}\left\{\hat{K}_{\alpha(n-r)}^{2} \hat{C}_{2(n-r)}^{2}-\hat{\triangle}_{2(n-r)}^{*} / \hat{\triangle}_{(n-r)}\right\} \\
& \min \cdot \operatorname{MSE}\left(d_{1}^{(1)}\right)=\min \cdot \operatorname{MSE}\left(d_{2}^{(1)}\right) \\
= & \hat{\theta}^{*} s_{0(n-r)}^{2}\left[1-\hat{\rho}_{02(n-r)}^{2}-\left\{\hat{\lambda}_{003(n-r)} \hat{\rho}_{02(n-r)}-\hat{\lambda}_{102(n-r)}\right\}^{2} / \hat{\triangle}_{(n-r)}\right]
\end{aligned}
$$

and

$$
\min . \operatorname{MSE}\left(d_{1(1)}^{(1)}\right)=\min \cdot \operatorname{MSE}\left(d_{2(1)}^{(1)}\right)=\hat{\theta}^{*} s_{0(n-r)}^{2}\left(1-\hat{\rho}_{02(n-r)}^{2}\right)
$$

where

$$
\operatorname{MS} E\left(\hat{R}_{\alpha(n-r)}\right)=\hat{\theta}^{*} \hat{R}_{\alpha(n-r)}^{2}\left[\hat{C}_{0(n-r)}^{2}+\alpha \hat{C}_{2(n-r)}^{2}\left\{\alpha-\hat{K}_{01(n-r)}\right\}\right] .
$$




\section{$3.2 \quad$ Strategy II}

We consider the situation when information on variables $y_{0}$ and $y_{1}$ cannot be obtained for $r$ units while population mean $\hat{Y}_{2}$ and variance $S_{2}^{2}$ of the auxiliary variable $y_{2}$ are known. We propose the following family of estimators of $R_{(\alpha)}$ as

$$
d_{3}=\hat{R}_{\alpha(n-r)} \phi(u, v)
$$

where $\phi(u, v)$ is a function of $(u, v)$ such that $\phi(1,1)=1$ and satisfies certain conditions similar to those for $f(\cdot)$ in $d_{1}$. Up to terms of order $o\left(n^{-1}\right)$, the bias and MSE of $d_{3}$ are respectively given by

$$
\begin{gathered}
B\left(d_{3}\right)=B\left(\hat{R}_{\alpha(n-r)}\right)+\left(\frac{\theta R_{(\alpha)}}{2}\right)\left[2 K_{(\alpha)} C_{2}^{2} \phi_{1}(1,1)+2 d_{(\alpha)} \phi_{2}(1,1)+C_{2}^{2} \phi_{11}(1,1)+\right. \\
\left.\left(\lambda_{004}-1\right) \phi_{22}(1,1)+2 \lambda_{003} C_{2} \phi_{12}(1,1)\right]
\end{gathered}
$$

and

$$
\begin{aligned}
\operatorname{MSE}\left(d_{3}\right) & =\operatorname{MSE}\left(\hat{R}_{\alpha(n-r)}\right)+\theta R_{(\alpha)}^{2}\left[C_{2}^{2} \phi_{1}^{2}(1,1)+\left(\lambda_{004}-1\right) \phi_{2}^{2}(1,1)\right. \\
& \left.+2 \lambda_{003} C_{2} \phi_{1}(1,1) \phi_{2}(1,1)+K_{(\alpha)} C_{2}^{2} \phi_{1}(1,1)+2 d_{(\alpha)} \phi_{2}(1,1)\right]
\end{aligned}
$$

The bias and MSE of an estimator belonging to the proposed family $d_{3}$ can be easily obtained from (3.2) and (3.3) respectively. The MSE of $d_{3}$ is minimized for

$$
\phi_{1}(1,1)=A \text { and } \phi_{2}(1,1)=B
$$

Thus the resulting minimum MSE of $d_{3}$ is given by

$$
\min \cdot \operatorname{MSE}\left(d_{3}\right)=\operatorname{MSE}\left(\hat{R}_{\alpha(n-r)}\right)-\theta R_{(\alpha)}^{2} B_{(\alpha)} .
$$

where $\operatorname{MSE}\left(\hat{R}_{\alpha(n-r)}\right)$ is given by (3.5). Thus we state the following theorem:

Theorem 3.5. Upto term of order $n^{-1}$,

$$
\operatorname{MSE}\left(d_{3}\right) \geq\left[\operatorname{MSE}\left(\hat{R}_{\alpha(n-r)}\right)-\theta R_{(\alpha)}^{2} B_{(\alpha)}\right]
$$

with equality holding if $\phi_{1}(1,1)=A$ and $\phi_{2}(1,1)=B$.

A family of estimators wider than $d_{3}$ is defined by

$$
d_{4}=\Phi\left(\hat{R}_{\alpha(n-r)}, u, v\right)
$$


where $\Phi\left(\hat{R}_{\alpha(n-r)}, u, v\right)$ is a function of $\left(\hat{R}_{\alpha(n-r)}, u, v\right)$ such that $\Phi(D)=R_{(\alpha)}$ and $\Phi_{1}(D)=1$. The bias and MSE of $d_{4}$ to the first degree of approximation, are respectively given by

$$
\begin{aligned}
B\left(d_{4}\right) & =B\left(\hat{R}_{\alpha(n-r)}\right)+\left(\frac{\theta}{2}\right)\left[2 R_{(\alpha)} K_{(\alpha)} C_{2}^{2} \Phi_{12}(D)+2 R_{(\alpha)} d_{(\alpha)} \Phi_{13}+C_{2}^{2} \Phi_{22}(D)+\right. \\
& \left.+\left(\lambda_{004}-1\right) \Phi_{33}(D)+2 \lambda_{003} C_{2} \Phi_{23}(D)\right]
\end{aligned}
$$

and

$$
\begin{aligned}
\operatorname{MSE}\left(d_{4}\right) & =\operatorname{MSE}\left(\hat{R}_{\alpha(n-r)}\right)+\theta\left[C_{2}^{2} \Phi_{2}^{2}(D)+\left(\lambda_{004}-1\right) \Phi_{3}^{2}(D)\right. \\
& \left.+2 \lambda_{003} C_{2} \Phi_{2}(D) \Phi_{3}(D)+2 R_{(\alpha)} K_{(\alpha)} C_{2}^{2} \Phi_{2}(D)+2 R_{(\alpha)} d_{(\alpha)} \Phi_{3}(D)\right]
\end{aligned}
$$

The MSE of $d_{4}$ is minimized for

$$
\Phi_{2}(D)=R_{(\alpha)} A=A^{*} \text { (say), and } \Phi_{3}(D)=R_{(\alpha)} B=B^{*} \text { (say). }
$$

and the resulting minimum MSE of $d_{4}$ is given by

$$
\min \cdot \operatorname{MSE}\left(d_{4}\right)=\left[\operatorname{MSE}\left(\hat{R}_{\alpha(n-r)}\right)-\theta R_{(\alpha)}^{2} B_{(\alpha)}\right] .
$$

It follows from (3.47) that to the firt degree of approximation, the minimum MSE of $d_{4}$ is equal to that of $d_{3}$ (i.e. $\left.\min \cdot \operatorname{MSE}\left(d_{4}\right)=\min \cdot \operatorname{MSE}\left(d_{3}\right)\right)$ and is not reduced. The difference type estimator, $d_{4(1)}=\hat{R}_{\alpha(n-r)}+\alpha_{1}(u-1)+\alpha_{2}(v-1)$, is a member of the family $d_{4}$, but not of $d_{3}$.

Remark 3.6. The following classes of estimators of $R_{(\alpha)}$ :

$$
\begin{array}{cc}
d_{4(1)}^{(1)}=\hat{R}_{\alpha(n-r)} \phi(u), & \text { (only } \bar{Y}_{2} \text { of } y_{2} \text { is known) } \\
d_{4(2)}^{(1)}=\hat{R}_{\alpha(n-r)} \phi(v), & \left(\text { only } S_{2}^{2} \text { of } y_{2}\right. \text { is known) } \\
d_{4(3)}^{(1)}=\Phi\left(\hat{R}_{\alpha(n-r)}, u\right), & \text { (only } \bar{Y}_{2} \text { of } y_{2} \text { is known) } \\
d_{4(4)}^{(1)}=\Phi\left(\hat{R}_{\alpha(n-r)}, v\right), & \text { (only } S_{2}^{2} \text { of } y_{2} \text { is known) }
\end{array}
$$

where $\phi(u), \phi(v), \phi\left(\hat{R}_{\alpha(n-r)}, u\right)$ and $\phi\left(\hat{R}_{\alpha(n-r)}, v\right)$ are the function of $u, v,\left(\hat{R}_{\alpha(n-r)},\right)$ and $\left(\hat{R}_{\alpha(n-r)}, v\right)$ respectively such that $\phi(1)=1$ and $\phi\left(R_{(\alpha)}, 1\right)=$ $R_{(\alpha)}$ which implies $\phi_{1}\left(R_{(\alpha)}, 1\right)=1$. The biases and MSEs of these classes of estimators can be easily obtained from (3.44) and (3.45) respectively for suitable values of the derivatives. 
Remark 3.7. The following families of estimators of population mean $\bar{Y}_{0}$ :

$$
\begin{gathered}
d_{4(1)}^{(0)}=\bar{y}_{0(n-r)} \phi(u, v), \\
d_{4(2)}^{(0)}=\Phi\left(\bar{y}_{0(n-r)} u, v\right), \\
d_{4(3)}^{(0)}=\bar{y}_{0(n-r)} \phi(u), \\
d_{4(4)}^{(0)}=\bar{y}_{0(n-r)} \phi(v), \\
d_{4(5)}^{(0)}=\Phi\left(\bar{y}_{0(n-r)}, u\right)
\end{gathered}
$$

and

$$
d_{4(6)}^{(0)}=\Phi\left(\bar{y}_{0(n-r)}, v\right)
$$

where $\phi(u, v), \quad \Phi\left(\bar{y}_{0(n-r)}, u, v\right), \phi(u), \phi(v), \Phi\left(\bar{u}_{0(n-r)}, u\right)$, and $\Phi\left(\bar{y}_{0(n-r)}, v\right)$ are the functions of $(u, v),\left(\bar{y}_{0(n-r)}, u, v\right),(u),(v),\left(\bar{y}_{0(n-r)}, u\right)$, and $\left(\bar{y}_{0(n-r)}, v\right)$ such that $\phi(1,1)=1, \phi(1)=1, \Phi\left(\bar{Y}_{0}, 1\right)=\bar{Y}_{0}$ which implies that $\Phi_{1}\left(\bar{Y}_{0}, 1\right)=1$, and $\Phi\left(D_{0}\right)=\bar{Y}_{0}$ which implies $\Phi\left(D_{0}\right)=1$. The biases and mean squared errors of these estimators can be obtained from (3.44) and (3.45) by putting $\alpha=0$ and suitable values of derivatives. The minimum MSE of $d_{4(1)}^{(0)}\left(\right.$ or $\left.d_{4(2)}^{(0)}\right), d_{4(3)}^{(0)}$ (or $\left.d_{4(5)}^{(0)}\right)$, and $d_{4(4)}^{(0)}\left(\right.$ or $\left.d_{4(6)}^{(0)}\right)$ respectively are

$$
\begin{aligned}
\min . \operatorname{MSE}\left(d_{4(1)}^{(0)}\right)= & \min \cdot \operatorname{MSE}\left(d_{4(2)}^{(0)}\right)=\theta S_{0}^{2}\left[1-\rho_{02}^{2}-\frac{\left(\lambda_{003} \rho_{02}-\lambda_{102}\right)^{2}}{\triangle}\right] \\
& +\left(\theta^{*}-\theta\right) S_{0}^{2},
\end{aligned}
$$

$$
\min \cdot \operatorname{MSE}\left(d_{4(3)}^{(0)}\right)=\min \cdot \operatorname{MSE}\left(d_{4(5)}^{(0)}\right)=\theta S_{0}^{2}\left(1-\rho_{02}^{2}\right)+\left(\theta^{*}-\theta\right) S_{0}^{2},
$$

and

$$
\min \cdot \operatorname{MSE}\left(d_{4(4)}^{(0)}\right)=\min \cdot \operatorname{MSE}\left(d_{4(6)}^{(0)}\right)=\theta S_{0}^{2}\left[1-\frac{\lambda_{102}^{2}}{\left(\lambda_{004}-1\right)}\right]+\left(\theta^{*}-\theta\right) S_{0}^{2} .
$$

Theorem 3.6. Upto terms of order $n^{-1}$,

(i) $\operatorname{MSE}\left(d_{4(1)}^{(0)}\right.$ or $\left.d_{4(2)}^{(0)}\right) \geq \theta S_{0}^{2}\left[1-\rho_{02}^{2}-\frac{\left(\lambda_{003} \rho_{02}-\lambda_{102}\right)^{2}}{\triangle}\right]+\left(\theta^{*}-\theta\right) S_{0}^{2}$ with equality holding if $\phi_{1}(1,1)=A_{11}$ and $\phi_{2}(1,1)=B_{11}$ for $d_{4(1)}^{(0)}$, and if $\Phi_{2}\left(D_{0}\right)=\bar{Y}_{0} A_{11}$ and $\Phi_{3}\left(D_{0}\right)=\bar{Y}_{0} B_{11}$ for $d_{4(2)}^{(0)}$. 
(ii) $\operatorname{MSE}\left(d_{4(3)}^{(0)}\right.$ or $\left.d_{4(5)}^{(0)}\right) \geq \theta S_{0}^{2}\left(1-\rho_{02}^{2}\right)+\left(\theta^{*}-\theta\right) S_{0}^{2}$

with equality holding if $\phi_{1}(1)=-K_{02}$ for $d_{4(3)}^{(0)}$, and if $\Phi_{2}\left(\bar{Y}_{0}, 1\right)=-K_{02} \bar{Y}_{0}$ for $d_{4(5)}^{(0)}$.

(iii) $\operatorname{MSE}\left(d_{4(4)}^{(0)}\right.$ or $\left.d_{4(6)}^{(0)}\right) \geq \theta S_{0}^{2}\left[1-\frac{\lambda_{102}^{2}}{\left(\lambda_{004}-1\right)}\right]+\left(\theta^{*}-\theta\right) S_{0}^{2}$ with equality holding if $\phi_{2}(1)=-\lambda_{102} C_{0} /\left(\lambda_{004}-1\right)$ for $d_{4(4)}^{(0)}$, and if $\phi_{3}\left(\bar{Y}_{0}, 1\right)=-\lambda_{102} C_{0} \bar{Y}_{0} /\left(\lambda_{004}-1\right)$ for $d_{4(6)}^{(0)}$.

It is to be noted that the estimator

$$
d_{4(7)}^{(0)}=\bar{y}_{0(n-r)}-\alpha_{2} \bar{Y}_{2}(u-1)
$$

of population mean $\bar{Y}_{0}$, suggested by Singh, Joarder and Tracy (2000) is a member of the family $d_{4(5)}^{(0)}\left(\right.$ or $\left.d_{4(2)}^{(0)}\right)$ at $(3.56)$ (or (3.53)) but not of $d_{4(3)}^{(0)}$ at (3.54), where $\alpha_{2}$ is a constant. The minimum MSE of $d_{4(7)}^{(0)}$ is the same as given by $(3.59)$.

\subsubsection{Estimators with Estimated Optimum Values}

The consistent estimators of optimum values $\phi_{1}(1,1)$ and $\phi_{2}(1,1)$ based on sample data at hand, are

$$
\hat{\phi}_{1}(1,1)=\hat{A}_{1} \text { (say) and } \hat{\phi}_{2}(1,1)=\hat{B}_{1}(\text { say }) .
$$

Thus the resulting family of estimators of $R_{(\alpha)}$ is defined as

$$
d_{3}^{*}=\hat{R}_{\alpha(n-r)} \phi^{*}\left(u, v, \hat{A}_{1}, \hat{B}_{1}\right)
$$

where $\phi^{*}(\cdot)$ is a function of $\left(u, v, \hat{A}_{1}, \hat{B}_{1}\right)$ such that $\phi^{*}(Z)=1, \phi_{1}^{*}(Z)=A, \phi_{2}^{*}(Z)=$ $B, \phi_{3}^{*}(Z)=0$, and $\phi_{4}^{*}(Z)=0$. It can be shown, to the first degree of approximation, that $\operatorname{MSE}\left(d_{3}^{*}\right)=\min \cdot \operatorname{MSE}\left(d_{3}\right)$ where $\min \cdot \operatorname{MSE}\left(d_{3}\right)$ is given by (3.42). Further a family of estimators (based on estimated optimum values) of $R_{(\alpha)}$ wider than $d_{3}^{*}$ is defined by

$$
d_{4}^{*}=\Phi^{*}\left(\hat{R}_{\alpha(n-r)}, u, v, \hat{A}_{1}^{*}, \hat{B}_{1}^{*}\right)
$$

where $\Phi^{*}\left(\hat{R}_{\alpha(n-r)}, u, v, \hat{A}_{1}^{*}, \hat{B}_{1}^{*}\right)$ is a function of $\left(\hat{R}_{\alpha(n-r)}, u, v, \hat{A}_{1}^{*}, \hat{B}_{1}^{*}\right)$ such that $\Phi^{*}\left(Z^{*}\right)=R_{(\alpha)}, \Phi_{1}^{*}\left(Z^{*}\right)=1, \Phi_{2}^{*}(\Psi)=\left.\frac{\partial \Phi^{*}(\bullet)}{\partial u}\right|_{\psi}=A^{*}, \Phi_{3}^{*}\left(Z^{*}\right)=B^{*}, \Phi_{4}^{*}\left(Z^{*}\right)=0$, 
and $\Phi_{5}^{*}\left(Z^{*}\right)=0$. It can be proved, to the first degree of approximation, that $\operatorname{MSE}\left(d_{4}^{*}\right)=\min \cdot \operatorname{MSE}\left(d_{4}\right)$, where $\min \cdot \operatorname{MSE}\left(d_{4}\right)$ is given by (3.47). Similarly various families of estimators of $R_{(\alpha)}$ based on estimated optimum values can be defined. Consistent estimators for estimating the minimum MSEs of various families are given in the following theorems.

Theorem 3.7. Estimators for estimating the minimum MSE of $d_{3}$ (or MSE of $\left.d_{3}^{*}\right), d_{4(1)}^{(0)}\left(\right.$ or $\left.d_{4(2)}^{(0)}\right), d_{4(3)}^{(0)}\left(\right.$ or $\left.d_{4(5)}^{(0)}\right)$ and $d_{4(4)}^{(0)}\left(\right.$ or $\left.d_{4(6)}^{(0)}\right)$ are, respectively, given by

$$
\begin{aligned}
\min \cdot \operatorname{MSE}\left(d_{3}\right) & =\operatorname{MSE}\left(d_{3}^{*}\right)=\operatorname{MSE}\left(\hat{R}_{\alpha(n-r)}\right) \\
& \left.-\theta \hat{R}_{\alpha(n-r)}^{2}\left\{\hat{K}_{(\alpha)}^{* 2} \hat{C}_{2}^{2}+\frac{\left(\hat{K}_{(\alpha)}^{*} \hat{\lambda}_{003} \hat{C}_{2}-\hat{d}_{\alpha(n-r)}\right)^{2}}{\hat{\lambda}_{004}-\hat{\lambda}_{003}^{2}-1}\right\}\right],
\end{aligned}
$$

$\min \cdot \operatorname{MSE}\left(d_{4(1)}^{(0)}\right)=\min \cdot \operatorname{MSE}\left(d_{4(2)}^{(0)}\right)=$

$$
\begin{aligned}
& \times \theta s_{0(n-r)}^{2}\left[1-\hat{\rho}_{0(n-r)}^{2}-\frac{\left(\hat{\lambda}_{003} \hat{\rho}_{02(n-r)}-\hat{\lambda}_{102(n-r)}\right)^{2}}{\hat{\lambda}_{004}-\hat{\lambda}_{003}^{2}-1}\right] \\
+ & \left(\frac{1}{n \hat{q}+2 \hat{p}}-\frac{1}{n}\right) s_{0(n-r)}^{2},
\end{aligned}
$$

$\min \cdot \operatorname{MS} E\left(d_{4(3)}^{(0)}\right)=\min \cdot \operatorname{MSE}\left(d_{4(5)}^{(0)}\right)=\left(\frac{1}{n \hat{q}+2 \hat{p}}-\frac{1}{n}\right) s_{0(n-r)}^{2}+\theta s_{0(n-r)}^{2}\left(1-\hat{\rho}_{02(n-r)}^{2}\right)$ and

$$
\begin{aligned}
& \min \cdot \operatorname{MSE}\left(d_{4(4)}^{(0)}\right)=\min \cdot \operatorname{MSE}\left(d_{4(6)}^{(0)}\right)=\theta s_{0(n-r)}^{2}\left\{1-\frac{\hat{\lambda}_{102(n-r)}^{2}}{\left(\hat{\lambda}_{004}-1\right)}\right\} \\
& +\left(\frac{1}{n \hat{q}+2 \hat{p}}-\frac{1}{n}\right) s_{\alpha(n-r)}^{2}
\end{aligned}
$$

\subsection{Strategy III}

We again consider the situation when information on study variables $y_{0}$ and $y_{1}$ cannot be obtained for $r$ units while information on the auxiliary variable $y_{2}$ is obtained for all the sample units. But the population mean $\bar{Y}_{2}$ and variance $S_{2}^{2}$ of the auxiliary character $y_{2}$ are not known. Under these circumstances, we define the following family of estimators of $R_{(\alpha)}$ as

$$
d_{5}=\hat{R}_{\alpha(n-r)} a\left(u^{*}, v^{*}\right)
$$


where $a\left(u^{*}, v^{*}\right)$ is a function of $u^{*}$ and $v^{*}$ such that $a(1,1)=1$ and satisfies certain regularity conditions as given in (3.1). To terms of order $n^{-1}$, the bias and MSE of $d_{5}$ are respectively given by

$$
\begin{aligned}
B\left(d_{5}\right) & =B\left(\hat{R}_{\alpha(n-r)}\right)+\left(\theta^{*}-\theta\right) \frac{R_{(\alpha)}}{2}\left[C_{2}^{2} a_{11}(1,1)+\left(\lambda_{004}-1\right) a_{22}(1,1)\right. \\
& \left.+2 \lambda_{003} C_{2} a_{12}(1,1)+2 K_{(\alpha)} C_{2}^{2} a_{1}(1,1)+2 d_{(\alpha)} a_{2}(1,1)\right]
\end{aligned}
$$

and

$$
\begin{aligned}
\operatorname{MSE}\left(d_{5}\right) & =\operatorname{MSE}\left(\hat{R}_{\alpha(n-r)}\right)+R_{\alpha(n-r)}^{2}\left(\theta^{*}-\theta\right)\left[C_{2}^{2} a_{1}^{2}(1,1)+\left(\lambda_{004}-1\right) a_{2}^{2}(1,1)\right. \\
& \left.+2 \lambda_{003} C_{2} a_{1}(1,1) a_{2}(1,1)+2 K_{(\alpha)} C_{2}^{2} a_{1}(1,1)+2 d_{(\alpha)} a_{2}(1,1)\right]
\end{aligned}
$$

The $\operatorname{MSE}\left(d_{5}\right)$ is minimized for

$$
a_{1}(1,1)=A \text { and } a_{2}(1,1)=B
$$

Thus the resulting (minimum) MSE of $d_{5}$ is given by

$\min \cdot \operatorname{MSE}\left(d_{5}\right)=\operatorname{MSE}\left(\hat{R}_{\alpha(n-r)}\right)-\left(\theta^{*}-\theta\right) R_{(\alpha)}^{2} B_{(\alpha)}=\min \cdot \operatorname{MSE}\left(d_{1}\right)+\theta R_{(\alpha)}^{2} B_{(\alpha)}$

where $\min \operatorname{MSE}\left(d_{1}\right)$ is given in (3.7). Thus we have the following theorem:

Theorem 3.8. Up to terms of order $n^{-1}, \operatorname{MSE}\left(d_{5}\right) \geq \min \cdot \operatorname{MSE}\left(d_{1}\right)+\theta R_{(\alpha)}^{2} B_{(\alpha)}$ with equality holding if $\alpha_{1}(1,1)=A$ and $\alpha_{2}(1,1)=B$.

Further we define a wider family of estimators for $R_{(\alpha)}$ as

$$
d_{6}=A\left(\hat{R}_{\alpha(n-r)}, u^{*}, v^{*}\right)
$$

where $A\left(\hat{R}_{\alpha(n-r)}, u^{*}, v^{*}\right)$ is a function of $\left(\hat{R}_{\alpha(n-r)}, u^{*}, v^{*}\right)$ such that $A(D)=R_{(\alpha)}$ and $A_{1}(D)=1$. To the first degree of approximation the bias of MSE of $d_{6}$ are respectively given by

$$
\begin{aligned}
B\left(d_{6}\right) & =B\left(\hat{R}_{\alpha(n-r)}\right)+\frac{\left(\theta^{*}-\theta\right)}{2}\left[C_{2}^{2} A_{22}(D)+\left(\lambda_{004}-1\right) A_{33}(D)+2 \lambda_{003} C_{2} A_{23}(D)\right. \\
& \left.+2 R_{(\alpha)} C_{2}^{2} K_{(\alpha)} A_{12}(D)+2 R_{(\alpha)} d_{(\alpha)} A_{13}(D)\right]
\end{aligned}
$$

and

$$
\begin{aligned}
\operatorname{MSE}\left(d_{6}\right)= & \operatorname{MSE}\left(\hat{R}_{\alpha(n-r)}\right)+\left(\theta^{*}-\theta\right)\left[C_{2}^{2} A_{2}^{2}(D)+\left(\lambda_{004}-1\right)\right. \\
& \left.A_{3}^{2}(D)+2 \lambda_{003} C_{2} A_{2}(D) A_{3}(D)+2 R_{(\alpha)} C_{2}^{2} K_{(\alpha)} A_{2}(D)+2 R_{(\alpha)} d_{(\alpha)} A_{3}(D)\right] .
\end{aligned}
$$


The $\operatorname{MSE}\left(d_{6}\right)$ is minimized for

$$
A_{2}(D)=R_{(\alpha)} A=A^{*} \text { and } A_{3}(D)=R_{(\alpha)} B=B^{*} .
$$

Thus the resulting minimum MSE of $d_{6}$ is given by

$$
\min \cdot \operatorname{MSE}\left(d_{6}\right)=\min \cdot \operatorname{MSE}\left(d_{5}\right)
$$

where $\min \operatorname{MSE}\left(d_{5}\right)$ is given by (3.67). Thus we see that the family of estimators $d_{6}$ is wider than $d_{5}$ but its minimum MSE is the same as that of $d_{5}$ and is not reduced. It is to be noted that the difference type estimator:

$$
d_{6(1)}=\hat{R}_{\alpha(n-r)}+\alpha_{1}\left(u^{*}-1\right)+\alpha_{2}\left(v^{*}-1\right)
$$

is a member of $d_{6}$, but not of $d_{5}$.

Remark 3.8. The following families of estimators of $R_{(\alpha)}$ :

$d_{6(1)}^{(1)}=\hat{R}_{\alpha(n-r)} a\left(u^{*}\right) ; d_{6(2)}^{(1)}=\hat{R}_{\alpha(n-r)} a\left(v^{*}\right) ; d_{6(3)}^{(1)}=A\left(\hat{R}_{\alpha(n-r)}, u^{*}\right) ; d_{6(4)}^{(1)}=\left(A \hat{R}_{\alpha(n-r)}, v^{*}\right)$

may be identified as member of the family $d_{6}$ and their biases and MSEs can be obtained from (3.69) and (3.70) respectively, where $a\left(u^{*}\right), a\left(v^{*}\right), A\left(\hat{R}_{\alpha(n-r)}, u^{*}\right)$ and $A\left(\hat{R}_{\alpha(n-r),}, v^{*}\right)$ are the functions of $u^{*}, v^{*},\left(\hat{R}_{\alpha(n-r)}, u^{*}\right)$ and $\left(\hat{R}_{\alpha(n-r)}, v^{*}\right)$ such that $a(1)=1$ and $A\left(R_{(\alpha)}, 1\right)=R_{(\alpha)}$ which implies $A_{1}\left(R_{(\alpha)}, 1\right)=1$.

Remark 3.9. If we set $\alpha=0$ in $d_{5}, d_{6}, d_{(j)}^{(1)} ; j=1,2,3,4$, we get the families of estimators for population mean $\bar{Y}_{0}$ as

$$
\begin{aligned}
& d_{6(1)}^{(0)}=\bar{y}_{0(n-r)} a\left(u^{*}, v^{*}\right) ; d_{6(2)}^{(0)}=A\left(\bar{y}_{0(n-r)}, u^{*}, v^{*}\right) ; d_{6(3)}^{(0)}=\bar{y}_{0(n-r)} a\left(u^{*}\right) \\
& d_{6(4)}^{(0)}=\bar{y}_{0(n-r)} a\left(v^{*}\right) ; d_{6(5)}^{(0)}=A\left(\bar{y}_{0(n-r)}, u^{*}\right) ; \text { and } d_{6(6)}^{(0)}=A\left(\bar{y}_{0(n-r)}, v^{*}\right)
\end{aligned}
$$

where $a\left(u^{*}, v^{*}\right), A\left(\bar{y}_{0(n-r)}, u^{*}, v^{*}\right), \alpha\left(u^{*}\right), \alpha\left(v^{*}\right), A\left(\bar{y}_{0(n-r)}, u^{*}\right), A\left(\bar{y}_{0(n-r)}, v^{*}\right)$ are the functions of $\left(u^{*}, v^{*}\right),\left(\bar{y}_{0(n-r)}, u^{*}, v^{*}\right),\left(\bar{y}_{0(n-r)}, u^{*}\right)$ and $\left(\bar{y}_{0(n-r)}, v^{*}\right)$ such that $a(1,1)=1, A\left(D_{0}\right)=\bar{Y}_{0}$ which implies $A_{1}\left(D_{0}\right)=1, a(1)=1$, and $A\left(\bar{Y}_{0}, 1\right)=\bar{Y}_{0}$ which implies that $A_{1}\left(\bar{Y}_{0}, 1\right)=1$. Putting $\alpha=0$ and suitable values of derivatives in (3.65) and (3.70), the biases and MSEs of these estimators can be easily obtained.

The minimum MSEs of the estimators $d_{(j)}^{(1)} ; j=1,2,3,4,5,6$ are given by $\min \cdot \operatorname{MSE}\left(d_{6(1)}^{(0)}\right)=\min \cdot \operatorname{MSE}\left(d_{6(2)}^{(0)}\right)=S_{0}^{2}\left[\theta^{*}-\left(\theta^{*}-\theta\right)\left\{\rho_{02}^{2}+\frac{\left(\lambda_{003} \rho_{02}-\lambda_{102}\right)^{2}}{\triangle}\right\}\right]$ 


$$
\min \cdot \operatorname{MSE}\left(d_{6(3)}^{(0)}\right)=\min \cdot \operatorname{MSE}\left(d_{6(5)}^{(0)}\right)=S_{0}^{2}\left[\theta^{*}-\left(\theta^{*}-\theta\right)\left(1-\rho_{02}^{2}\right)\right]
$$

and

$$
\min \cdot \operatorname{MSE}\left(d_{6(4)}^{(0)}\right)=\min \cdot \operatorname{MSE}\left(d_{6(6)}^{(0)}\right)=S_{0}^{2}\left[\theta^{*}-\left(\theta^{*}-\theta\right)\left\{1-\frac{\lambda_{102}^{2}}{\lambda_{004}-1}\right\}\right] .
$$

Thus we establish the following theorem:

Theorem 3.9. Upto terms of order $n^{-1}$

(i) $\operatorname{MSE}\left(d_{6(1)}^{(0)}\right.$ or $\left.d_{6(2)}^{(0)}\right) \geq S_{0}^{2}\left[\theta^{*}-\left(\theta^{*}-\theta\right)\left\{\rho_{02}^{2}+\frac{\left(\lambda_{003} \rho_{02}-\lambda_{102}\right)^{2}}{\triangle}\right\}\right]$ which equality holding if $a_{1}(1,1)=A$ and $a_{2}(1,1)=B$ for $d_{6(1)}^{(0)}$, and if $A_{2}\left(D_{0}\right)=\bar{Y}_{0} A_{11}$ and $A_{3}\left(D_{0}\right)=\bar{Y}_{0} B_{11}$ for $d_{6(2)}^{(0)}$.

(ii) $\operatorname{MSE}\left(d_{6(3)}^{(0)}\right.$ or $\left.d_{6(5)}^{(0)}\right) \geq S_{0}^{2}\left[\theta^{*}-\left(\theta^{*}-\theta\right)\left(1-\rho_{02}^{2}\right)\right]$

with equality holding if $a_{1}(1)=-K_{02}$ for $d_{6(3)}^{(0)}$, and if $A_{2}\left(\bar{Y}_{2}, 1\right)=-\bar{Y}_{0} K_{02}$ for $d_{6(5)}^{(0)}$.

(iii) $\operatorname{MSE}\left(d_{6(4)}^{(0)}\right.$ or $\left.d_{6(6)}^{(0)}\right) \geq S_{0}^{2}\left[\theta^{*}-\left(\theta^{*}-\theta\right)\left\{1-\frac{\lambda_{102}^{2}}{\lambda_{004}-1}\right\}\right]$

with equality holding if $a_{2}(1)=-\lambda_{102} C_{0} /\left(\lambda_{004}-1\right)$ for $d_{6(4)}^{(0)}$, and if $A_{3}\left(\bar{Y}_{0}, 1\right)=$ $-\bar{Y}_{0} \lambda_{102} C_{0} /\left(\lambda_{004}-1\right)$ for $d_{6(6)}^{(0)}$. It is to be mentioned that the estimator $d_{6(7)}^{(0)}=$ $\bar{y}_{0(n-r)}+\alpha_{3} \bar{y}_{2}\left(u^{*}-1\right)$ is a member of $d_{6(5)}^{(0)}\left(\right.$ or $\left.d_{6(2)}^{(0)}\right)$ but not of $d_{6(3)}^{(0)}\left(\right.$ or $\left.d_{6(1)}^{(0)}\right)$. It can be shown to the first degree of approximation that

$$
\min \cdot \operatorname{MSE}\left(d_{6(7)}^{(0)}\right)=\min \cdot \operatorname{MSE}\left(d_{6(5)}^{(0)}\right)
$$

where min.MSE $\left(d_{6(5)}^{(0)}\right)$ is given by (3.74). The estimator $d_{6(7)}^{(0)}$ is due to Singh, Joarder and Tracy (2000).

\subsubsection{Estimators Based on Estimated Parameters}

The estimated optimum values of $a_{1}(1,1)$ and $a_{2}(1,1)$ based on sample observations are respectively given by

$$
\hat{a}_{1}(1,1)=\hat{A}_{2} \text { (say) and } \hat{a}_{2}(1,1)=\hat{B}_{2} \text { (say). }
$$

Thus we define a family of estimators (based on estimated optimum values) for $R_{(\alpha)}$ as

$$
d_{5}^{*}=\hat{R}_{\alpha(n-r)} a^{*}\left(u^{*}, v^{*}, \hat{A}_{2}, \hat{B}_{2}\right)
$$


where $a^{*}(\cdot)$ is a function of $\left(u^{*}, v^{*}, \hat{A}_{2}, \hat{B}_{2}\right)$ such that $a^{*}(Z)=1, a_{1}^{*}(Z)=A$, $a_{2}^{*}(Z)=B, a_{3}^{*}(Z)=0$, and $\alpha_{4}^{*}(Z)=0$. It can be shown, to the first degree of approximation, that

$$
\operatorname{MSE}\left(d_{5}^{*}\right)=\min \cdot \operatorname{MSE}\left(d_{5}\right)
$$

where $\min \cdot \operatorname{MSE}\left(d_{5}\right.$ is given by $(3.67)$.

Further a wider class of estimators (based on estimated optimum parameters) of $R_{(\alpha)}$ is defined by

$$
d_{6}^{*}=A^{*}\left(\hat{R}_{\alpha(n-r)}, u^{*}, v^{*}, \hat{A}_{2}^{*}, \hat{B}_{2}^{*}\right)
$$

where $A^{*}(\cdot)$ is a function of $\left(\hat{R}_{\alpha(n-r)}, u^{*}, v^{*}, \hat{A}_{2}^{*}, \hat{B}_{2}^{*}\right)$ such that $A^{*}(Q)=R_{(\alpha)}, A_{1}^{*}(Q)=$ $1, A_{2}^{*}(Q)=A^{*}, A_{3}^{*}(Q)=B^{*}, A_{4}^{*}(Q)=0, A_{5}^{*}(Q)=0$ with $\hat{A}_{2}^{*}=\hat{R}_{\alpha(n-r)} \hat{A}_{2}$ and $\hat{B}_{2}^{*}=\hat{R}_{\alpha(n-r)} \hat{B}_{2}$. It is easy to verify that to the first degree of approximation,

$$
\operatorname{MSE}\left(d_{6}^{*}\right)=\min \cdot \operatorname{MSE}\left(d_{5}^{*}\right)=\min \cdot \operatorname{MSE}\left(d_{6}\right)
$$

where min.MSE $\left(d_{5}\right)$ is given in (3.67). The estimator

$$
d_{6(1)}^{*}=\hat{R}_{\alpha(n-r)}+\hat{A}_{2}^{*}\left(u^{*}-1\right)+\hat{B}_{2}^{*}\left(v^{*}-1\right)
$$

is a particular member of the family $d_{6}^{*}$. The MSE of $d_{6}^{*}$, to terms of order $n^{-1}$, is same as that of $d_{5}^{*}$.

Similar many other families of estimators based on estimated optimum values can be defined with their approximate MSEs formulae.

Now, we give below the consistent estimators of the minimum MSEs of the family of estimators.

Theorem 3.10. Estimators of the minimum MSE of the family of estimators $d_{5}\left(\right.$ or $\left.d_{6}\right)$ (or MSE of $d_{5}^{*}$ or $\left.d_{6}^{*}\right), d_{6(j)}^{(0)}, j=1,2, d_{6(3)}^{(0)}\left(\right.$ or $\left.d_{6(5)}^{(0)}\right)$ and $d_{6(4)}^{(0)}\left(\right.$ or $\left.d_{6(6)}^{(0)}\right)$ are, respectively, given by

$$
\begin{gathered}
\min \operatorname{MS} E\left(d_{j}\right)=\operatorname{MSE}\left(d_{j}^{*}\right)=\min \cdot \operatorname{MSE}\left(\hat{R}_{\alpha(n-r)}\right)-\left(\hat{\theta}^{*}-\theta\right) \hat{R}_{\alpha(n-r)}^{2} \hat{B}_{\alpha(n-r)}, \\
\min . \operatorname{MSE}\left(d_{6(1)}^{(0)}\right)=\min \operatorname{MSSE}\left(d_{6(2)}^{(0)}\right)=s_{0(n-r)}^{2}\left[\hat{\theta}^{*}-\left(\hat{\theta}^{*}-\theta\right)\left(\hat{\rho}_{02(n-r)}^{2}+\hat{C}_{\alpha(n-r)}\right)\right], \\
\min . \operatorname{MS} E\left(d_{6(3)}^{(0)}\right)=\min \operatorname{MSE}\left(d_{6(5)}^{(0)}\right)=s_{0(n-r)}^{2}\left[\theta+\left(\hat{\theta}^{*}-\theta\right)\left(1-\hat{\rho}_{02(n-r)}^{2}\right)\right],
\end{gathered}
$$


and $\min \cdot \operatorname{MS} E\left(d_{6(4)}^{(0)}\right)=\min \operatorname{MS} E\left(d_{6(6)}^{(0)}\right)=s_{0(n-r)}^{2}\left[\theta+\left(\hat{\theta}^{*}-\theta\right)\left\{1-\hat{\lambda}_{102(n-r)}^{2} /\left(\hat{\lambda}_{004}-1\right)\right\}\right]$ where $j=5,6$ and min.MŜE $\left(\hat{R}_{\alpha(n-r)}\right)$ is given in Theorem 3.4.

\section{Efficiency Comparisons}

From (3.5), (3.7), (3.23) and (3.24), we have

$$
\begin{gathered}
\operatorname{MSE}\left(\hat{R}_{\alpha(n-r)}\right)-\min \cdot \operatorname{MSE}\left(d_{1}\right)=\theta^{*} R_{(\alpha)}^{2} B_{(\alpha)} \geq 0, \\
\operatorname{MSE}\left(\hat{R}_{\alpha(n-r)}\right)-\min \cdot \operatorname{MS} E\left(d_{1(1)}\right)=\theta^{*} R_{(\alpha)}^{2} C_{2}^{2} K_{(\alpha)}^{2} \geq 0, \\
\operatorname{MSE}\left(\hat{R}_{\alpha(n-r)}\right)-\min \cdot \operatorname{MSE}\left(d_{1(2)}\right)=\theta^{*} R_{(\alpha)}^{2} d_{(\alpha)}^{2} /\left(\lambda_{004}-1\right) \geq 0, \\
\operatorname{MSE}\left(d_{1(1)}\right)-\min \cdot \operatorname{MSE}\left(d_{1}\right)=\theta^{*} R_{(\alpha)}^{2} \frac{\triangle_{2}^{2}}{\triangle} \geq 0,
\end{gathered}
$$

$\min . \operatorname{MSE}\left(d_{1(2)}\right)-\min \cdot \operatorname{MSE}\left(d_{1}\right)=\theta^{*} R_{(\alpha)}^{2} \frac{\left\{K_{(\alpha)} C_{2}\left(\lambda_{004}-1\right)-d_{(\alpha)} \lambda_{003}\right\}^{2}}{\left(\lambda_{004}-\lambda_{003}^{2}-1\right)\left(\lambda_{004}-1\right)} \geq 0$.

Thus we have the following inequalities

$$
\min \cdot \operatorname{MSE}\left(d_{1}\right) \leq \min \cdot \operatorname{MSE}\left(d_{1(1)}\right) \leq \operatorname{MSE}\left(\hat{R}_{\alpha(n-r)}\right)
$$

and

$$
\min \cdot \operatorname{MSE}\left(d_{1}\right) \leq \min \cdot \operatorname{MSE}\left(d_{1(2)}\right) \leq \operatorname{MSE}\left(\hat{R}_{\alpha(n-r)}\right)
$$

It is well known that

$$
\operatorname{Var}\left(\bar{y}_{0(n-r)}\right)=\theta^{*} S_{0}^{2}
$$

From (3.27), (3.32), (3.33) and (4.8) we have

$$
\begin{gathered}
\operatorname{Var}\left(\bar{y}_{0(n-r)}\right)-\min \cdot \operatorname{MSE}\left(d_{1(1)}^{(1)}\right)=\theta^{*} S_{0}^{2} \rho_{02}^{2} \geq 0 \\
\operatorname{Var}\left(\bar{y}_{0(n-r)}\right)-\min \cdot \operatorname{MSE}\left(d_{1(2)}^{(1)}\right)=\theta^{*} S_{0}^{2} \lambda_{102}^{2} /\left(\lambda_{004}-1\right) \geq 0 \\
\min . \operatorname{MSE}\left(d_{1(1)}^{(1)}\right)-\min \cdot \operatorname{MSE}\left(d_{1}^{(1)}\right)=\theta^{*} S_{0}^{2}\left(\lambda_{003} \rho_{02}-\lambda_{102}\right)^{2} / \triangle \geq 0 \\
\min . \operatorname{MSE}\left(d_{1(2)}^{(1)}\right)-\min \cdot \operatorname{MSE}\left(d_{1}^{(1)}\right)=\theta^{*} S_{0}^{2}\left(\rho_{02}\left(\lambda_{004}-1\right)-\lambda_{102} \lambda_{003}\right)^{2} / \triangle \geq 0 .
\end{gathered}
$$


Thus we have the following inequalities:

$$
\min \cdot \operatorname{MSE}\left(d_{1}^{(1)}\right) \leq \min \cdot \operatorname{MSE}\left(d_{1(1)}^{(1)}\right) \leq \operatorname{Var}\left(\bar{y}_{0(n-r)}\right)
$$

and

$$
\min \cdot \operatorname{MSE}\left(d_{1}^{(1)}\right) \leq \min \cdot \operatorname{MSE}\left(d_{1(2)}^{(1)}\right) \leq \operatorname{Var}\left(\bar{y}_{0(n-r)}\right) .
$$

It follows from (4.13) and (4.14) that the estimator $d_{1}^{(1)}$ is more efficient than $\bar{y}_{0(n-r)}, d_{1(1)}^{(1)}, d_{1(2)}^{(1)}$ and Singh, Joarder and Tracy (2000) estimator $d_{2(3)}^{(1)}$.

Similarly following inequalities can easily be proved:

$$
\min \cdot \operatorname{MSE}\left(d_{3}\right) \leq \min \cdot \operatorname{MSE}\left(d_{4(1)}^{(1)}\right) \leq \operatorname{MSE}\left(\hat{R}_{\alpha(n-r)}\right)
$$

and

$$
\min \cdot \operatorname{MSE}\left(d_{3}\right) \leq \min \cdot \operatorname{MSE}\left(d_{4(2)}^{(1)}\right) \leq \operatorname{MSE}\left(\hat{R}_{\alpha(n-r)}\right) .
$$

Thus from (4.15) and (4.16) it follows that the suggested family of estimators $d_{3}\left(\right.$ or $d_{3}^{*}$ or $\left.d_{4}\right)$ is better than the conventional estimator $\hat{R}_{\alpha(n-r)}, d_{4(1)}^{(1)}$ ( or $\left.d_{4(3)}^{(1)}\right)$ and or $d_{4(2)}^{(1)}\left(\right.$ or $\left.d_{4(4)}^{(1)}\right)$.

From (3.58), (3.59), (3.60) and (4.8) following inequalities hold:

$$
\min \cdot \operatorname{MSE}\left(d_{4(1)}^{(0)}\right) \leq \min \cdot \operatorname{MSE}\left(d_{4(3)}^{(0)}\right) \leq \operatorname{Var}\left(\bar{y}_{0(n-r)}\right)
$$

and

$$
\min \cdot \operatorname{MSE}\left(d_{4(1)}^{(0)}\right) \leq \min \cdot \operatorname{MSE}\left(d_{4(4)}^{(0)}\right) \leq \operatorname{Var}\left(\bar{y}_{0(n-r)}\right) .
$$

Thus the proposed estimator $d_{4(1)}^{(0)}\left(\right.$ or $\left.d_{4(2)}^{(0)}\right)$ is better than usual unbiased estimator $\bar{y}_{0(n-r)}, d_{4(3)}^{(0)}\left(\right.$ or $\left.d_{4(5)}^{(0)}\right), d_{4(4)}^{(0)}\left(\right.$ or $\left.d_{4(6)}^{(0)}\right)$ and Singh, Joarder and Tracy (2000) estimator $d_{4(7)}^{(0)}$.

Further it can be easily proved that

$$
\min \cdot \operatorname{MSE}\left(d_{5}\right) \leq \min \cdot \operatorname{MSE}\left(d_{6(1)}^{(1)}\right) \leq \operatorname{MSE}\left(\hat{R}_{\alpha(n-r)}\right)
$$

and

$$
\min \cdot \operatorname{MSE}\left(d_{5}\right) \leq \min \cdot \operatorname{MSE}\left(d_{6(2)}^{(1)}\right) \leq \operatorname{MSE}\left(\hat{R}_{\alpha(n-r)}\right)
$$

Thus the proposed family of estimators $d_{5}\left(\right.$ or $\left.d_{6}\right)$ is more efficient than $\hat{R}_{0(n-r)}, d_{6(1)}^{(1)}\left(\right.$ or $\left.d_{6(3)}^{(1)}\right)$, and $d_{6(2)}^{(1)}\left(\right.$ or $\left.d_{6(4)}^{(1)}\right)$.

From (3.73), (3.74), (3.75) and (4.8) it is easy to see that

$$
\min \cdot \operatorname{MSE}\left(d_{6(1)}^{(0)}\right) \leq \min \cdot \operatorname{MSE}\left(d_{6(3)}^{(0)}\right) \leq \operatorname{Var}\left(\bar{y}_{0(n-r)}\right)
$$


and

$$
\min \cdot \operatorname{MSE}\left(d_{6(1)}^{(0)}\right) \leq \min \cdot \operatorname{MSE}\left(d_{6(4)}^{(0)}\right) \leq \operatorname{Var}\left(\bar{y}_{0(n-r)}\right) .
$$

It follows from (4.21) and (4.22) that the estimator $d_{6(1)}^{(0)}\left(\right.$ or $\left.d_{6(2)}^{(1)}\right)$ is more efficient than the estimator $\bar{y}_{0(n-r)}, d_{6(3)}^{(0)}\left(\right.$ or $\left.d_{6(5)}^{(0)}\right), d_{6(4)}^{(0)}\left(\right.$ or $\left.d_{6(6)}^{(1)}\right)$ and the estimator $d_{6(7)}^{(0)}$ due to Singh, Joarder and Tracy (2000). From (3.7), (3.42) and (3.67) we have

$$
\min \cdot \operatorname{MSE}\left(d_{3}\right)-\min \cdot \operatorname{MSE}\left(d_{1}\right)=\left(\theta^{*}-\theta\right) R_{(\alpha)}^{2} \triangle_{2}^{2} / \triangle \geq 0
$$

and

$$
\min \cdot \operatorname{MSE}\left(d_{5}\right)-\min \cdot \operatorname{MSE}\left(d_{3}\right)=\theta R_{(\alpha)}^{2} B_{(\alpha)} \geq 0
$$

It follows from the above inequalities that the proposed family $d_{1}$ (or $d_{1}^{*}$ ) is the best among all the estimators discussed in the present investigation.

\section{Conclusion}

The article has suggested families of estimators of the parameter $R_{(\alpha)}$ in presence of random non-response together with their biases and mean squared errors. The proposed families include several classes of estimators of the parameters $R$ whose biases and mean squared errors can be obtained easily. Thus the proposal of families of estimators is justified as they unify several results. It has been shown that the proposed families are better than usual estimators as well as several other families of estimators. It is interesting to note that the families of estimators based on 'estimated optimum values' have same mean squared errors up to first degree of approximation, as that of optimum estimators in the families which depend upon the unknown population parameters. It also provides several families of estimators of popultion mean $\bar{Y}_{0}$ which are better than conventional unbiased estimator and Singh, Joarder and Tracy (2000) estimators. Finally it is found that the proposed family $d_{1}\left(\right.$ or $\left.d_{1}^{*}\right)$ or $d_{2}$ (or $\left.d_{2}^{*}\right)$ is the best in the sense that it has least minimum MSE.

\section{Appendix: General Notations}

The following notations have been used throughout the paper: $N:$ Number of units in the population; $n$ : number of units in the sample; $r$ : number of sampling units on which information could not be obtained due to random non-response $(r=0,1,2, \ldots,(n-2)) ; p:$ the probability of non-response among the $(n-2)$ 
possible values of non-response; $q=(1-p) ; \theta=\left(\frac{1}{n}-\frac{1}{N}\right) ; \theta^{*}=\left(\frac{1}{n q+2 p}-\frac{1}{N}\right) ; \delta=$ $\left(\theta^{*}-\theta\right)$; SRSWOR: Simple random without replacement sample $(n-r)$ : Number of remaining units in the sample treated as SRSWOR sample from the population; $\mathrm{B}(\cdot)$ : Bias of $(\cdot) ; \operatorname{MSE}(\cdot)$ : mean squared error.

For variates $y_{0}, y_{1}$, and $y_{2}$ in the population: $\bar{Y}_{i}=N^{-1} \sum_{j=1}^{N} y_{i j}$ : the population mean of the $i$ th variate $y_{i}(i=0,1,2): R_{(\alpha)}=\bar{Y}_{0} / \bar{Y}_{1}^{\alpha},\left(\bar{Y}_{1} \neq 0\right)$ : the population parameter under study; $\alpha$ : being a scalar takes values 0,1 and $-1 ; C_{i}$ : the population coefficient of variation $(\mathrm{CV})$ of the variate $y_{i}(i=0,1,2) ; \rho_{i l}=$ $S_{i l} /\left(S_{i} S_{l}\right), i \neq l$ : the population correlation coefficient between the variates $y_{i}$ and $y_{l}(i \neq l=0,1,2)$;

$$
\begin{aligned}
& (N-1) S_{i l}=\sum_{j=1}^{N}\left(y_{i j}-\bar{Y}_{i}\right) ;\left(y_{l j}-\bar{Y}_{l}\right) K_{i l}=\rho_{i l} C_{i} / C_{l}(i \neq l=0,1,2) ; \\
& A_{(\alpha)}=\left[C_{0}^{2}+\alpha C_{1}^{2}\left(\alpha-2 K_{01}\right)\right] ; \\
& K_{(\alpha)}=\left(K_{02}-\alpha K_{12}\right) ; d_{(\alpha)}=\left(\lambda_{102}-\alpha \lambda_{012} C_{1}\right) ; A_{1}=\triangle_{1} /\left(\triangle C_{2}\right) ; \\
& A^{*}=R_{(\alpha)} A ; B=\triangle_{2} / \triangle ; B^{*}=R_{(\alpha)} B ; \\
& A_{11}=\triangle_{11} /\left(\triangle C_{2}\right) ; B_{11}=\triangle_{22} / \triangle ; \triangle_{1}=\left[d_{(\alpha)} \lambda_{003}-K_{(\alpha)}\left(\lambda_{004}-1\right) C_{2}\right] ; \\
& \triangle_{2}=\left[K_{(\alpha)} \lambda_{003} C_{2}-d_{(\alpha)}\right] ; \\
& \triangle=\left(\lambda_{004}-\lambda_{003}^{2}-1\right)>0 ; \triangle_{11}=\left[\lambda_{101} C_{0}-K_{02}\left(\lambda_{004}-1\right) C_{2}\right] ; \\
& \triangle_{22}=\left[K_{02} \lambda_{003} C_{2}-\lambda_{102} C_{0}\right] ; B_{(\alpha)}=\left(K_{(\alpha)}^{2} C_{2}^{2}+\triangle B^{2}\right) ; \\
& K=-K_{02} ; k^{*}=-\bar{Y}_{0} K_{02}=-\beta_{02} \bar{Y}_{2} ; \beta_{02}=S_{02} / S_{2}^{2} ; \\
& \lambda_{m_{1} m_{2} m_{3}}=\mu_{m_{1} m_{2} m_{3}} /\left(\mu_{200}^{m_{1} / 2} \mu_{020}^{m_{2} / 2} \mu_{002}^{m_{3} / 2}\right) ; \\
& (N-1)_{m_{1} m_{2} m_{3}}=\sum_{j=1}^{N}\left(y_{0 j}-\bar{Y}_{0}\right)^{m_{1}}\left(y_{1 j}-\bar{Y}_{1}\right)^{m_{2}}\left(y_{2 j}-\bar{Y}_{2}\right)^{m_{3}} \\
& \left(m_{1}, m_{2}, m_{3}\right) \text { non-negative integers. }
\end{aligned}
$$

For the variates $y_{0}, y_{1}$ and $y_{2}$ in the sample: $\bar{y}_{i}=n^{-1} \sum_{j=1}^{n} y_{i j},(i=0,1,2) ; \hat{R}_{(\alpha)}=$ $\bar{y}_{0} / \bar{y}_{1}^{\alpha},\left(\bar{y}_{1} \neq 0\right) ;(n-1) s_{i}^{2}=\sum_{j=1}^{n}\left(y_{i j}-\bar{y}_{i}\right)^{2} ;(n-r) \bar{y}_{i(n-r)}=\sum_{j=1}^{n-r} y_{i j}$; $(n-r-1) s_{i(n-r)}^{2}=\sum_{j=1}^{n-r}\left(y_{i j}-\bar{y}_{i(n-r)}\right)^{2}$ : the conditional unbiased estimator of $S_{i}^{2}(i=0,1,2,) ; \hat{R}_{\alpha(n-r)}=\bar{y}_{0(n-r)} / \bar{y}_{1(n-r)}^{\alpha},\left(\bar{y}_{1(n-r)} \neq 0\right) ; u=\bar{y}_{2} / \bar{Y}_{2} ; \nu=s_{2}^{2} / S_{2}^{2}$;

$u_{(n-r)}=\bar{y}_{2(n-r)} / \bar{Y}_{2} ; \nu_{(n-r)}=s_{2(n-r)}^{2} / S_{2}^{2} ; u^{*}=\bar{y}_{2(n-r)} / \bar{y}_{2} ; \nu^{*}=s_{2(n-r)}^{2} / s_{2}^{2}$;

$\hat{A}=\hat{\triangle}_{1(n-r)} /\left(\hat{\triangle}_{(n-r)} C_{2}\right) ; \hat{B}=\hat{\triangle}_{2(n-r)} / \hat{\triangle}_{(n-r)} ; \hat{A}^{*}=\hat{R}_{\alpha(n-r)} \hat{A} ; \hat{B}^{*}$

$=\hat{R}_{\alpha(n-r)} \hat{B} ; \hat{A}_{1}=\hat{\triangle}_{1} /\left(\hat{\triangle} C_{2}\right) ; \hat{A}_{2}=\hat{\triangle}_{1}^{*} /\left(\hat{\triangle} C_{2}\right) ; \hat{B}_{1}=\hat{\triangle}_{2} / \hat{\triangle}$

$\hat{B}_{2}=\hat{\triangle}_{2}^{*} / \hat{\triangle} ; \hat{A}_{1}^{*}=\hat{R}_{\alpha(n-r)} \hat{A}_{1} ; \hat{B}_{1}^{*}=\hat{R}_{\alpha(n-r)} \hat{B}_{1} ; \hat{A}_{2}^{*}=\hat{R}_{\alpha(n-r)} \hat{A}_{2} ; \hat{B}_{2}^{*}=\hat{R}_{\alpha(n-r)} \hat{B}_{2} ; \hat{\triangle}=$ $\left(\hat{\lambda}_{004}-\hat{\lambda}_{003}^{2}-1\right)>0$ 


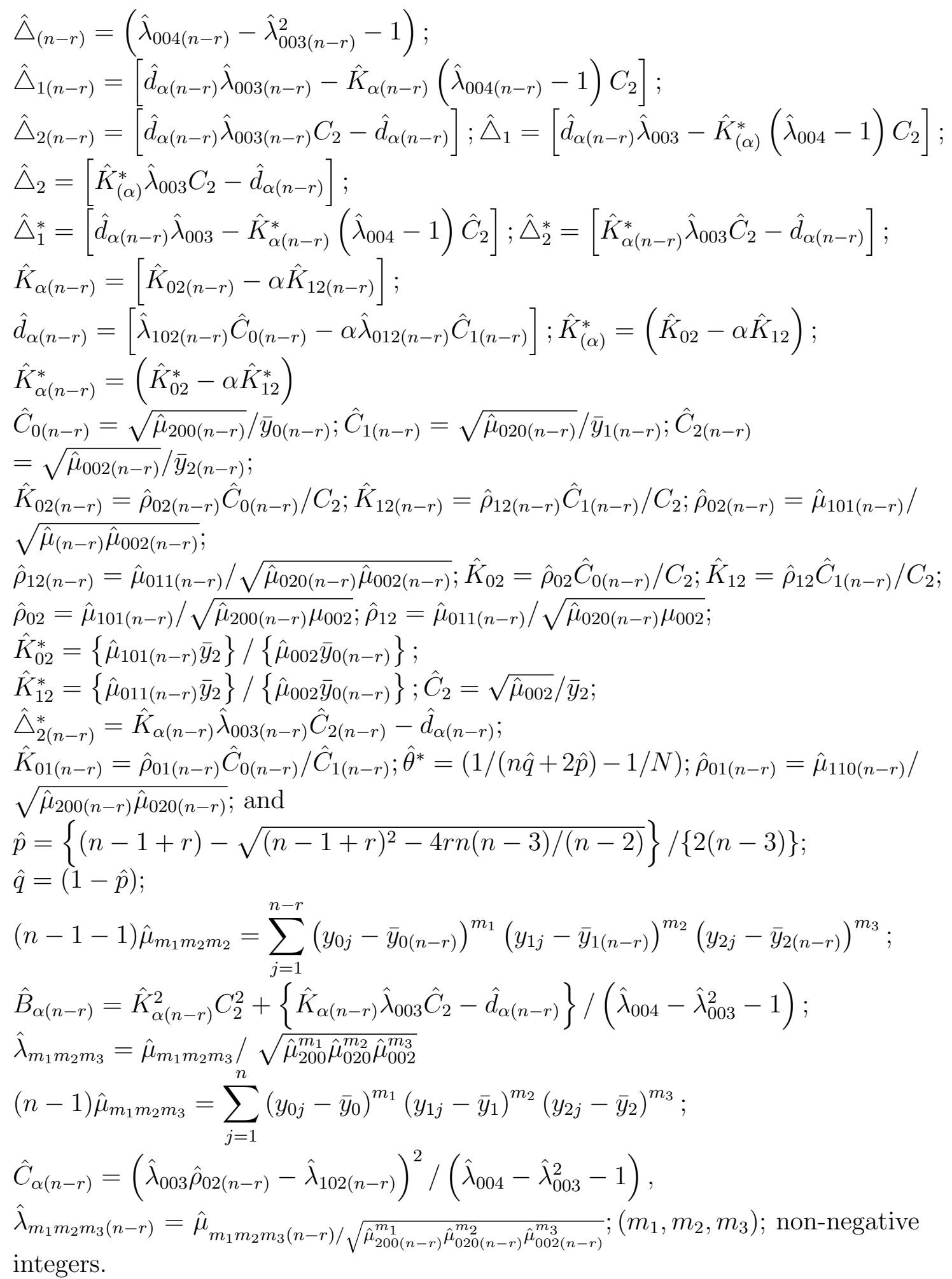

In addition, we have used the following:

- $H_{1}\left(R_{(\alpha)}, 1\right)$ and $H_{2}\left(R_{(\alpha)}, 1\right)$ : denote the first order partial derivatives of 
the function $H\left(\hat{R}_{(\alpha)}, u\right)$ with respect to (w.r.t) $\hat{R}(\alpha)$ and $u$ respectively about the point $\left(R_{(\alpha)}, 1\right)$;

- $H_{i j}\left(R_{(\alpha)}, 1\right),(i, j=1,2)$ : denote the second order partial derivatives of the function $H\left(\hat{R}_{(\alpha)}, u\right)$ about the point $\left(R_{(\alpha)}, 1\right)$;

- $t_{1}(1,1)$ and $t_{2}(1,1)$ : denote the first order partial derivatives of the function $t(u, v)$ w.r.t. $u$ and $\nu$ respectively about the point $(1,1)$;

- $t_{i j}(1,1),(i, j=1,2)$ : denote the second order partial derivatives of the function $t(u, \nu)$ about the point $(1,1)$;

- $T_{1}(D), T_{2}(D)$ and $T_{3}(D):$ denote the first order partial derivatives of the of the function $T\left(\hat{R}_{(\alpha)}, u, \nu\right)$ w.r.t. $\hat{R}_{(\alpha)}, u$ and $\nu$ respectively about the point $D=\left(R_{(\alpha)}, 1,1\right)$;

- $T_{i j}(D),(i, j=1,2)$ : denote the second order partial derivatives of the function $T\left(\hat{R}_{(\alpha)}, u, \nu\right)$ about the point $D$;

- $f_{1}(1,1)$ and $f_{2}(1,1)$ : denote the first order partial derivatives of the function $f\left(u_{(n-r)}, \nu_{(n-r)}\right)$ w.r.t. $u_{(n-r)}$ and $\nu_{(n-r)}$ respectively about the point $(1,1)$;

- $f_{i j}(1,1),(i, j=1,2)$ : denote the second order partial derivatives of the function $f\left(u_{(n-r)}, \nu_{(n-r)}\right)$ about the point $(1,1)$;

- $\left(f_{1}(1), f_{11}(1)\right)$ and $\left(f_{2}(1), f_{22}(1)\right)$ : denote the first and second order partial derivatives of the functions $f\left(u_{(n-r)}\right)$ and $f\left(\nu_{(n-r)}\right)$ with respect to $u_{(n-r)}$ and $\nu_{(n-r)}$ respectively about the point 'unity'.

- $h_{1}$ and $h_{11}(1)$ : denote the first and the second order partial derivatives of the function $h(u)$ about the point 'unity'.

- $F_{1}(D), F_{2}(D)$ and $F_{3}(D)$ : denote the first order partial derivatives of the function $F\left(\hat{R}_{(\alpha)}, u_{(n-r)}, \nu_{(n-r)}\right)$ w.r.t. $\hat{R}_{(\alpha)}, u_{(n-r)}$ and $\nu_{(n-r)}$ respectively about the point $D$;

- $F_{i j}(D),(i, j=1,2,3)$ : denote the second order partial derivatives of the function $F\left(\hat{R}_{(\alpha)}, u_{(n-r)}, \nu_{(n-r)}\right)$ about the point $D$;

- $F_{1}\left(R_{(\alpha)}, 1\right), F_{2}\left(R_{(\alpha)}, 1\right)$ and $F_{3}\left(R_{(\alpha)}, 1\right)$ : denote the first order partial derivatives of the functions $\left(F\left(\hat{R}_{\alpha(n-r)}, u_{(n-r)}\right), F\left(\hat{R}_{\alpha(n-r)}, \nu_{(n-r)}\right)\right)$ w.r.t. $\hat{R}_{\alpha(n-r)}, F\left(\hat{R}_{\alpha(n-r)}, u_{(n-r)}\right)$ w.r.t. $\quad u_{(n-r)}$, and $F\left(\hat{R}_{\alpha(n-r)}, u_{(n-r)}\right)$ w.r.t. $\nu_{(n-r)}$ respectively about the point $\left(R_{(\alpha)}, 1\right)$; 
- $F_{12}\left(R_{(\alpha)}, 1\right)$ and $F_{22}\left(R_{(\alpha)}, 1\right)$ : denote the second order partial derivatives of the function $F\left(\hat{R}_{\alpha(n-r)}, u_{(n-r)}\right)$ about the point $\left(R_{(\alpha)}, 1\right)$;

- $F_{13}\left(R_{(\alpha)}, 1\right)$ and $F_{33}\left(R_{(\alpha)}, 1\right)$ : denote the second order partial derivatives of the function $F\left(\hat{R}_{\alpha(n-r)}, v_{(n-r)}\right)$ about the point $\left(R_{(\alpha)}, 1\right)$;

- $f_{1}^{*}(Z), f_{2}^{*}(Z), f_{3}^{*}(Z)$ and $f_{4}^{*}(Z)$ : denote the first order partial derivatives of the function $f^{*}\left(u_{(n-r)}, \nu_{(n-r)}, \hat{A}, \hat{B}\right)$ w.r.t. $u_{(n-r)}, \nu_{(n-r)}, \hat{A}$ and $\hat{B}$ respectively about the point $Z=(1,1, A, B)$;

- $F_{1}^{*}\left(Q^{*}\right), F_{2}^{*}\left(Q^{*}\right), F_{3}^{*}\left(Q^{*}\right), F_{4}^{*}\left(Q^{*}\right)$ and $F_{5}^{*}\left(Q^{*}\right)$ : denote the first order partial derivatives of the function $F\left(\hat{R}_{\alpha(n-r)}, u_{(n-r)}, \nu_{(n-r)}, \hat{A}, \hat{B}\right)$ w.r.t.

$\hat{R}_{\alpha(n-r)}, u_{(n-r)}, \nu_{(n-r)}, \hat{A}^{*}$ and $\hat{B}^{*}$ respectively about the point $Q^{*}=\left(R_{(\alpha)}, 1,1, A^{*}, B^{*}\right) ;$

- $F_{1}\left(D_{0}\right), F_{2}\left(D_{0}\right)$ and $F_{3}\left(D_{0}\right)$ : denote the first order partial derivatives of the function $F\left(\bar{y}_{0(n-r)}, u_{(n-r)}, \nu_{(n-r)}\right)$ w.r.t. $\bar{y}_{0(n-r)}, u_{(n-r)}$ about $\nu_{(n-r)}$ and the point $D_{0}=\left(\bar{Y}_{0}, 1,1\right)$;

- $F_{i j}\left(D_{0}\right),(i, j=1,2,3)$ : denote the second order partial derivatives of the function $F\left(\bar{y}_{0(n-r)}, u_{(n-r)}, \nu_{(n-r)}\right)$ about the point $D_{0}=\left(\bar{Y}_{0}, 1,1\right)$;

- $F_{2}\left(\bar{Y}_{0}, 1\right)$ : denotes the first order partial derivative of the function $F\left(\bar{y}_{0(n-r)}, u_{(n-r)}\right)$ w.r.t. $u_{(n-r)}$ about the point $\left(\bar{Y}_{0}, 1\right)$ :

- $F_{i j}\left(\bar{Y}_{0}, 1\right),(i, j=1,2)$ : denote the second order partial derivatives of the function $F\left(\bar{y}_{0(n-r)}, u_{(n-r)}\right)$ about the point $\left(\bar{Y}_{0}, 1\right)$;

- $F_{3}\left(\bar{Y}_{0}, 1\right)$ : denotes the first order partial derivative of the function $F\left(\bar{y}_{0(n-r)}, v_{(n-r)}\right)$ w.r.t $\nu_{(n-r)}$ about the point $\left(\bar{Y}_{0}, 1\right)$;

- $F_{i j}\left(\bar{Y}_{0}, 1\right),(i, j=1,3)$ : denote the second order partial derivatives of the function $F\left(\bar{y}_{0(n-r)}, \nu_{(n-r)}\right)$ about the point $\left(\bar{Y}_{0}, 1\right)$;

- $\phi_{1}(1,1)$ and $\phi_{2}(1,1)$ : denote the first order partial derivatives of the function $\phi(u, \nu)$ w.r.t. $u$ and $\nu$ respectively about the point $(1,1)$;

- $\phi_{i j}(1,1),(i, j=1,2)$ : denote the second order partial derivatives of the function $\phi(u, \nu)$ about the point $(1,1)$;

- $\Phi_{1}(D), \Phi_{2}(D)$ and $\Phi_{3}(D)$ : denote the first order partial derivatives of the function $\Phi\left(\hat{R}_{\alpha(n-r)}, u, \nu\right)$ w.r.t. $\hat{R}_{\alpha(n-r)}, u$ and $\nu$ respectively about the point $D$; 
- $\Phi_{i j}(D),(i, j=12,3)$ : denote the second order partial derivatives of the function $\Phi\left(\hat{R}_{\alpha(n-r)}, u, \nu\right)$ about the point $D$;

- $\Phi_{1}\left(D_{0}\right), \Phi_{2}\left(D_{0}\right)$ and $\Phi_{3}\left(D_{0}\right)$ : denote the first order partial derivatives of the function $\Phi\left(\bar{y}_{0(n-r)}, u, v\right)$ w.r.t. $\bar{y}_{0(n-r)}, u$ and $\nu$ respectively about the point $D_{0}$;

- $\Phi_{1}\left(\bar{Y}_{0}, 1\right)$ : denotes the first order partial derivative of the functions $\Phi\left(\bar{y}_{0(n-r)}, u\right)$ and $\Phi\left(\bar{y}_{0(n-r)}, \nu\right)$ w.r.t. $\bar{y}_{0(n-r)}$, about the point $\left(\bar{Y}_{0}, 1\right)$;

- $\Phi_{2}\left(\bar{Y}_{0}, 1\right)$ : denotes the first order partial derivative of the functions $\Phi\left(\bar{y}_{0(n-r)}, u\right)$ w.r.t. $u$ about the point $\left(\bar{Y}_{0}, 1\right)$;

- $\Phi_{3}\left(\bar{Y}_{0}, 1\right)$ : denotes the first order partial derivative of the function $\Phi\left(\bar{y}_{0(n-r)}, \nu\right)$ w.r.t. $v$ about the point $\left(\bar{Y}_{0}, 1\right)$;

- $\Phi_{1}(1)$ and $\Phi_{2}(1)$ : denote the first order partial derivative of the function $\Phi(u)$ and $\Phi(\nu)$ w.r.t. $u$ and $\nu$ respectively about the point 'unity'.

- $\Phi_{1}\left(R_{(\alpha)}, 1\right)$ : denotes the first order partial derivative of the functions $\Phi\left(\hat{R}_{\alpha(n-r)}, u\right)$ and $\Phi\left(\hat{R}_{\alpha(n-r)}, \nu\right)$ w.r.t. $\hat{R}_{\alpha(n-r)}$ about the point $\left(R_{(\alpha)}, 1\right)$;

- $\Phi_{1}^{*}(Z), \Phi_{2}^{*}(Z), \Phi_{3}^{*}(Z)$ and $\Phi_{4}^{*}(Z)$ : denote the first order partial derivatives of the function $\Phi^{*}\left(u, \nu, \hat{A}_{1}^{*}, \hat{B}_{1}^{*}\right)$ w.r.t. $u, \nu, \hat{A}_{1}^{*}$ and $\hat{B}_{1}^{*}$ about the point $\left(1,1, A^{*}, B^{*}\right)=Z$;

- $\Phi_{1}^{*}\left(Z^{*}\right), \Phi_{2}^{*}\left(Z^{*}\right), \Phi_{3}^{*}\left(Z^{*}\right)$ and $\Phi_{4}^{*}\left(Z^{*}\right)$ : denote the first order partial derivatives of the function $\Phi^{*}\left(\hat{R}_{\alpha(n-r)}, u, \nu, \hat{A}_{1}^{*}, \hat{B}_{1}^{*}\right)$ w.r.t. $\hat{R}_{\alpha(n-r)}, u, \nu, \hat{A}_{1}^{*}$ and $\hat{B}_{1}^{*}$ about the point $\left(R_{(\alpha)}, 1,1, A^{*}, B^{*}\right)=Z^{*}$;

- $a_{1}(1,1)$ and $a_{2}(1,1):$ denote the first order partial derivatives of the function $a\left(u^{*}, \nu^{*}\right)$ w.r.t. $u^{*}$ and $\nu^{*}$ respectively about the point $(1,1)$;

- $a_{i j}(1,1), i, j=1,2:$ denotes the first order partial derivatives of the function $a\left(u^{*}, \nu^{*}\right)$ about the point $(1,1)$;

- $A_{1}(D), A_{2}(D)$ and $A_{3}(D)$ : denote the first order partial derivatives of the function $A\left(\hat{R}_{\alpha(n-r)}, u^{*}, \nu^{*}\right)$ w.r.t. $\hat{R}_{\alpha(n-r)}, u^{*}$ and $\nu^{*}$ respectively about the point $D$;

- $A_{i j}(D), i, j=1,2,3$ : denote the second order partial derivatives of the function $A\left(\hat{R}_{\alpha(n-r)}, u^{*}, \nu^{*}\right)$ about the point $D$; 
- $A_{1}\left(R_{(\alpha)}, 1\right)$ : denotes the first order partial derivative of the functions $A\left(\hat{R}_{\alpha(n-r)}, u^{*}\right)$ and $A\left(\hat{R}_{\alpha(n-r)}, \nu^{*}\right)$ with respect to $\hat{R}_{\alpha(n-r)}$ about the point $\left(R_{\alpha)}, 1\right)$;

- $A_{1}\left(D_{0}\right), A_{2}\left(D_{0}\right)$ and $A_{3}\left(D_{0}\right)$ : denote the first order partial derivatives of the function $A\left(\hat{y}_{0(n-r)}, u^{*}, \nu^{*}\right)$ w.r.t. $\bar{y}_{0(n-r)}, u^{*}$ and $\nu^{*}$ respectively about the point $D_{0}$;

- $A_{1}\left(\bar{Y}_{0}, 1\right)$ : denotes the first order partial derivative of the function $A\left(\bar{y}_{0(n-r)}, u^{*}\right)$ and $A\left(\bar{y}_{0(n-r)}, \nu^{*}\right)$ w.r.t. $\bar{y}_{0(n-r)}$ about the point $\left(\bar{Y}_{0}, 1\right)$;

- $A_{2}\left(\bar{Y}_{0}, 1\right)$ : denotes the first order partial derivative of the function $A\left(\bar{y}_{0(n-r)}, u^{*}\right)$ w.r.t. $u^{*}$ about the point $\left(\bar{Y}_{0}, 1\right)$;

- $A_{3}\left(\bar{Y}_{0}, 1\right)$ : denotes the first order partial derivative of the function $A\left(\bar{y}_{0(n-r)}, \nu^{*}\right)$ w.r.t. $\nu^{*}$ about the point $\left(\bar{Y}_{0}, 1\right)$;

- $a_{1}(1)$ : denotes the first order partial derivative of the function $a\left(u^{*}\right)$ w.r.t. $u^{*}$ about the point 'unity';

- $a_{2}(1)$ : denotes the first order partial derivative of the function $a\left(\nu^{*}\right)$ w.r.t. $\nu^{*}$ about the point 'unity';

- $a_{1}^{*}(Z), a_{2}^{*}(Z), a_{3}^{*}(Z)$ and $a_{4}^{*}(Z)$ : denote the first order partial derivatives of the function $a^{*}\left(u^{*}, \nu^{*}, \hat{A}_{2}, \hat{B}_{2}\right)$ w.r.t. $u^{*}, \nu^{*}, \hat{A}_{2}$ and $\hat{B}_{2}$ respectively about the point $Z$;

- $A_{1}^{*}\left(Q^{*}\right), A_{2}^{*}\left(Q^{*}\right), A_{3}^{*}\left(Q^{*}\right), A_{4}^{*}\left(Q^{*}\right)$ and $A_{5}^{*}\left(Q^{*}\right)$ : denote the first order partial derivatives of the function $A^{*}\left(\hat{R}_{\alpha(n-r)}, u^{*}, \nu^{*}, \hat{A}_{2}^{*}, \hat{B}_{2}^{*}\right)$ w.r.t. $\hat{R}_{\alpha(n-r)}, u^{*}, \nu^{*}, \hat{A}_{2}^{*}$ and $\hat{B}_{2}^{*}$ respectively about the point $Q^{*}$;

\section{Acknowledgements}

The authors are thankful to the Editor Prof. Enrique Castillo, an Associate Editor, and the two learned referees for valuable comments to bring the original manuscript in the present form. The third author, Anwar H. Joarder, acknowledges King Fahd University of Petroleum and Minerals, Dhahran, Saudi Arabia for providing excellent research facilities. 


\section{References}

[1] Heitjan, D.F. and Basu, S. (1996). Distinguishing 'Missing at Random' and 'Missing Completely at Random'. The American Statistician, 50, 207-213.

[2] Randles, R. (1982). On the asymptotic normality of statistics with estimated parameters. Annals of Statistics, 10(2), 462-474.

[3] Rao, J.N.K. and Pereira, N.P. (1968). On double ratio estimators. Sankhya, A, 30, 83-90.

[4] Ray, S.K. and Singh, R.K. (1985). Some estimators for the ratio and product of population parameters. J. Indian Soc. Agric. Statist., 37(1), 1-10.

[5] Rubin, D.B. (1976), Inference and missing data. Biometrika, 63(3), 581-592.

[6] Shah, S.M. and Shah, D.N. (1978). Ratio-cum-product estimator for estimating ratio (product) of two popultion parameters. Sankhya, C, 40(2), 156-166.

[7] Singh, M.P. (1965). On the estimation of ratio and product of population parameters. Sankhya, B. 27, 321-328.

[8] Singh, M.P. (1967). Ratio cum product method of estimation. Metrika, 12(1), $34-43$.

[9] Singh, H.P. (1986a). Estimation of ratio, product and mean using auxiliary information in sample surveyr. Aligarh Jour. Statist., 6, 32-44.

[10] Singh, H.P. (1986b). A generalized class of estimators of ratio, product and mean using supplementary information on an auxiliary character in PPSWR sampling scheme. Gujarat Statist. Review, 13(2), 1-30.

[11] Sihgh, H.P. (1988). On the estimation of ratio and product of two finite popultion means. Proceedings of the National Academy of Science of India, 58A, III, 399403.

[12] Singh, H.P. and Tracy, D.S. (2001). Estimation of population mean in presence of random non-response in sample surveys. Statistica, 231-248.

[13] Singh, S. and Joarder, A.H. (1998). Estimation of finite population variance using random non-response in survey sampling. Metrika, 47, 241-249.

[14] Singh, S., Joarder, A.H. and Tracy, D.S. (2000). Regression type estimators for random non-response in survey sampling. Statistics, LX, no.1, 39-44.

[15] Singh, S. and Singh, R. (1979). On random non-response in unequal sampling. Sankhya, 41, 27-37.

[16] Singh, S. and Singh, R. (1985). On random non-response in double sampling with difference estimator. Commun. Statist.-Simul. \& Compu., 14, 747-757.

[17] Singh, V.K., Singh, H.P., Singh, H.P. and Shukla, D. (1994a). A general class of chain estimators for ratio and product of two means of a finite population. Commun. Statist- Theory Meth, 23, 1341-1355.

[18] Singh, V.K., Singh, H.P. and Singh, H.P. (1994b). Estimation of ratio and product of two finite population means in two-phase sampling. J. Statist. Plnn. Inference, 41,163-171.

[19] Srivastava, S.K. (1971). A generalized estimator for the mean of a finite population using multiauxiliary information. J. Amer. Statist. Assoc., 66, 404-407.

[20] Srivastava, S.K. (1980). A class of estimators using auxiliary information in sample surveys. Canadian. J. Statist., 8, 253-254. 
[21] Srivastava, S.K. and Jhajj, H.S. (1981). A class of estimators of the population mean in survey sampling using auxiliary information. Biometrika, 68, 341-343.

[22] Srivastava, S.R., Srivastava, S.R., and Khare, B.B. (1989). Chain ratio tye estimators for ratio of two population means using auxiliary characters. Commun. Statist.,-Theory Meth, 18(10), 3917-3926.

[23] Toutenberg, H. and Srivastava, V.K. (1998). Estimation of ratio of popultion means in survey sampling when some observatins are missing. Metrika, 48, 177187.

[24] Tracy, D.S. and Osahan, S.S. (1994). Random non-response on study variable versus on study as well as auxiliary variables. Statistica, LIV, n.2, 163-168.

[25] Tripathi, T.P. (1980). A general class of estimators for poupultion ratio. Sankhya, C, 42, 63-75.

[26] Upadhyaya, L.N. and Singh, H.P. (1985). A class of estimators using auxiliary information for estimating ratio of two finite population means. Gujarat Statistical Review, 12(2), 7-16.

[27] Upadhyaya, L.N., Singh, H.P. and Vos, J.W.E. (1985). On the estimation of population means and ratios using supplementary information. Statistica Neerlandica, 39(3), 309-318. 